TRANSACTIONS OF THE

AMERICAN MATHEMATICAL SOCIETY

Volume 352, Number 9, Pages 4067-4094

S 0002-9947(00)02455-7

Article electronically published on April 17, 2000

\title{
THE MARKOV SPECTRA FOR FUCHSIAN GROUPS
}

\author{
L. YA. VULAKH
}

\begin{abstract}
Applying the Klein model $D^{2}$ of the hyperbolic plane and identifying the geodesics in $D^{2}$ with their poles in the projective plane, the author develops a method of determining infinite binary trees in the Markov spectrum for a Fuchsian group. The method is applied to a maximal group commensurable with the modular group and other Fuchsian groups.
\end{abstract}

\section{INTRODUCTION}

Let $f_{x}(u)=f_{x}\left(u_{1}, u_{2}\right)=x_{0} u_{1}^{2}+x_{1} u_{1} u_{2}+x_{2} u_{2}^{2}=x_{0}\left(u_{1}-\theta u_{2}\right)\left(u_{1}-\theta^{\prime} u_{2}\right)$ be an indefinite quadratic form with real coefficients and with discriminant $Q(x)=$ $x_{1}^{2}-4 x_{0} x_{2}$. Define $\nu\left(f_{x}\right)=\nu(x)=\inf \left|f_{x}\left(u_{1}, u_{2}\right)\right| Q(x)^{-1 / 2}$, the infimum being taken over all $\left(u_{1}, u_{2}\right) \in \mathbf{Z}^{2} /(0,0)$. The set $\mathcal{M}=\left\{\nu\left(f_{x}\right): x \in \mathbf{R}^{3}, Q(x)>0\right\}$ is called the Markov spectrum. In 1879, Markov 20 showed, by means of continued fractions, that the set $\mathcal{M} \cap(1 / 3, \infty)$ is discrete and it consists of the numbers $\left(9-4 / m^{-2}\right)^{-1 / 2}$, where $m$ runs through the set of all positive integers such that $\left(m, m_{1}, m_{2}\right)$ is a solution of the Diophantine equation $m^{2}+m_{1}^{2}+m_{2}^{2}=3 m m_{1} m_{2}$. This result is closely related with the subject of Diophantine approximations (see e.g. 4] or [9]). Another development, started by Frobenius (1913) and Remak (1924), led to a new proof of Markov's theorem using the properties of quadratic forms [4]. See [17, [12, 23], 21], 26] for other proofs of the original Markov theorem and its generalization. See also [19] and [9 for the history of the problem and for the known results on the structure of the Markov spectrum $\mathcal{M}$ below $1 / 3$.

Since $\left|f_{x}\left(u_{1}, u_{2}\right)\right| Q(x)^{-1 / 2}=\left|\theta-\theta^{\prime}\right|^{-1}$, to study the Markov spectrum we can associate with real numerical multiples of an indefinite quadratic form $f_{x}$ as above the geodesic $\gamma$ in the hyperbolic plane $H^{2}=\{z \in \mathbf{C}: \operatorname{Im} z>0\}$ whose endpoints $\theta$ and $\theta^{\prime}$ lie in $\mathbf{R}$. This more recent point of view led to many generalizations and extensions of the original Markov theorem. The most spectacular of these is related to the parametrization of the set of simple closed geodesics on some coverings of the modular surface $H^{2} / S L_{2}(\mathbf{Z})$ by the Markov spectrum above $1 / 3$ (see [6], [7, [3], 13, [14, [15], 18]).

In this paper, we shall use the Klein model of the hyperbolic plane. In particular, we identify the form $f_{x}$ with the point $x=\left(x_{0}, x_{1}, x_{2}\right)$ in the projective plane $P^{2}$. Then the interior of the conic $Q(x)=0$ can be considered as a model $D^{2}$ of the hyperbolic plane. If $f_{x}$ is indefinite, then the intersection of the polar of $x$ (see the definition below) with $D^{2}$ is a geodesic in $D^{2}$. The main emphasis will be

Received by the editors September 17, 1997 and, in revised form, August 25, 1998.

2000 Mathematics Subject Classification. Primary 11J06, 11 F06.

Key words and phrases. Diophantine approximation, projective geometry, hyperbolic geometry.

(C)2000 American Mathematical Society 
on determination of the reduction region $\mathcal{R}$ which is the set of extremal points $x \in P^{2}\left(x\right.$ is extremal if $\left|f_{x}\left(u_{1}, u_{2}\right)\right| \geq\left|x_{0}\right|$ for all $\left.\left(u_{1}, u_{2}\right) \in \mathbf{Z}^{2} /(0,0)\right)$. For any $x \in \mathcal{R}, \nu(x)=\left|x_{0} Q(x)^{-1 / 2}\right|$. Thus, it is easy to find the spectrum $\mathcal{M}$ if $\mathcal{R}$ is known. On the other hand, $\mathcal{R}$ is two-dimensional and its "projection" $\mathcal{M}$ is one-dimensional, and many structural features of the spectrum which are hidden in $\mathcal{M}$ can be revealed in $\mathcal{R}$. In particular, it will be shown in this paper that an infinite binary tree whose limit set lies on a certain conic in $P^{2}$ is one of the basic structures of $\mathcal{R}$ outside $D^{2}$. We first give the necessary definitions, state the problem in general for an $(n+1)$-dimensional quadratic space, and give the known examples of its solution. In the rest of the paper only the case of $n=2$ (the Klein model of the hyperbolic plane) will be discussed. Readers interested only in that case may skip the following definitions, statement of the problem in general, and Examples 1-4 below, and read 39, pages 12-13, instead.

Let $V$ be an $(n+1)$-dimensional vector space over $\mathbf{R}$. Let $Q: V \rightarrow \mathbf{R}$ be a regular quadratic form with associated symmetric bilinear form

$$
(x, y)=(Q(x+y)-Q(x-y)) / 4 \text {. }
$$

An $\mathbf{R}$-linear transformation $A$ from the quadratic space $(V, Q)$ into itself is called an isometry if $(A x, A y)=(x, y)$ for any $x, y \in V$. The set of all isometries of $(V, Q)$ will be denoted by $O(V)=O(V, Q)$. Two vectors $v, u \in V$ are said to be orthogonal if $(u, v)=0$. Let $U$ be a linear subspace of $V$. The linear subspace $U^{\perp}=\{v \in V:(v, U)=0\}$ of $V$ is the orthogonal complement to $U$ in $V$. Any $v \in V$ can be represented uniquely as $v=v_{1}+v_{2}$, where $v_{1} \in U, v_{2} \in U^{\perp}$. In that case we have the "Pythagorean theorem" $Q(v)=Q\left(v_{1}\right)+Q\left(v_{2}\right)$, and we say that $x_{1}=x_{U}$ is the projection of $x$ into $U$ (see e.g. [5]).

We shall say that a subspace $U$ of $V$ is regular if the restriction $Q_{U}$ of $Q$ to $U$ is, and that the signature of $U \subset V$ is $(s, t)$ if the signature of $Q_{U}$ is $(s, t)$. There are finitely many $O(V)$-orbits of regular subspaces $U \subset V$ (such orbits are called the Grassmanians of $V$ ). Any such orbit is uniquely determined by the signature $(s, t), s+t=\operatorname{dim} U$. In that case the signature of $Q_{U^{\perp}}$ is $(p-s, q-t)$, where $(p, q)$ is the signature of $Q$ and $p+q=n+1$.

This paper mainly concerns the classification of the regular subspaces of $V$ modulo the action of a discrete subgroup of $O(V)$. An important example of a discrete subgroup $G$ of $O(V)$ is the group $O(L)$, which is defined as follows. A lattice $L \subset V$ is said to be $Q$-integral if $(L, L) \subset \mathbf{Z}$. If $e_{0}, \ldots, e_{n}$ is a basis of an integral lattice $L$, then $Q\left(x_{0}, \ldots, x_{n}\right)=Q\left(x_{0} e_{0}+\ldots+x_{n} e_{n}\right)$ has integral coefficients. The group $O(L)=(g \in O(V): g(L)=L)$ is a discrete cofinite subgroup of $O(V)$.

Let $G$ be a discrete subgroup of $O(V)$. Let $w \in V$ be a fixed nonzero vector. For a subspace $U$ of $V$ we define

$$
\nu(U)=\nu(w, U)=\inf \left|Q\left(x_{U}\right)\right|
$$

the infimum being taken over all $x \in G w$, and

$$
\nu_{o}(U)=\nu_{o}(w, U)=\inf \left|Q\left(x_{U}\right)\right|
$$

the infimum being taken over all $x \in G w$ such that $Q\left(x_{U}\right) \neq 0$. It is clear that

$$
\nu(g U)=\nu(U), \quad g \in G \text {. }
$$

We shall denote by $\mathcal{M}(s, t)=\mathcal{M}_{w}(s, t)$ the set of all values of $\nu(U)$, where $U$ runs through the set of all linear subspaces of $V$ of signature $(s, t)$. The set $\mathcal{M}_{w}(s, t)$ will be called the spectrum of $(s, t)$ - minima with respect to $w$. Similarly, $\mathcal{M}_{o}(s, t)$, 
the spectrum of nonzero $(s, t)$-minima with respect to $w$, is defined as the set of values of $\nu_{o}(U)$.

A subspace $U$ of $V$ is said to be $w$-extremal if

$$
\nu(U)=\left|Q\left(w_{U}\right)\right|,
$$

and the set $\mathcal{R}_{w}(s, t)$ of all $w$-extremal subspaces of $V$ of signature $(s, t)$ will be called the $w$-reduction region of $(s, t)$-subspaces of $V$.

If a vector $w \in V$ is isotropic, i.e. $Q(w)=0$, then $Q\left(w_{1}\right)=-Q\left(w_{2}\right), w_{1} \in$ $U, w_{2} \in U^{\perp}$. Hence

$$
\nu(U)=\nu\left(U^{\perp}\right),
$$

and $U$ is $w$-extremal if and only if $U^{\perp}$ is. Thus, $\mathcal{M}_{w}(s, t)=\mathcal{M}_{w}(p-s, q-t)$ in that case. When $(s, t)=(n+1,0)$ or $(1, n)$ the $w$-reduction region of $(1,0)$ subspaces of $V$ is related to the Dirichlet region of $G$ in Euclidean or hyperbolic space with center at $w$ (see Example 1).

When the signature of $Q$ is $(n, 1)$, the spectrum $\mathcal{M}_{w}(1,1)$ is related to the problem of Diophantine approximation in Euclidean spaces (see Example 3).

Let $U, U^{\prime} \subset V$. If $U^{\prime}=g U$ for some $g \in G$, we say that subspaces $U$ and $U^{\prime}$ are $G$-equivalent. The $w$-reduction region $\mathcal{R}_{w}(s, t)$ and the spectrum $\mathcal{M}_{o}(s, t)$ can be used to classify the $G$-equivalence classes (or $G$-orbits) of subspaces of signature $(s, t)$ of $V$ (see Example 2).

Below, the known examples of classification of $O(V)$-orbits of regular subspaces $U \subset V$ modulo the action of a discrete subgroup $G$ of $O(V)$ are given.

Examples of reduction regions and spectra of minima.

Let $e_{1}, \ldots, e_{k}$ be an orthogonal basis of a subspace $U \subset V$. Then $x \in U$ can be represented as follows:

$$
x=\frac{\left(x, e_{1}\right)}{Q\left(e_{1}\right)} e_{1}+\ldots+\frac{\left(x, e_{k}\right)}{Q\left(e_{k}\right)} e_{k} .
$$

Thus, for $x \in V$,

$$
Q\left(x_{U}\right)=\frac{\left(x, e_{1}\right)^{2}}{Q\left(e_{1}\right)}+\ldots+\frac{\left(x, e_{k}\right)^{2}}{Q\left(e_{k}\right)} .
$$

In particular, when $k=1$,

$$
Q\left(x_{U}\right)=\frac{\left(x, e_{1}\right)^{2}}{Q\left(e_{1}\right)} .
$$

Let $U$ be a hyperbolic plane, i.e. the signature of $U$ is $(1,1)$. Let $e_{1}, e_{2}$ be a basis of $U$ such that $Q\left(e_{1}\right)=Q\left(e_{2}\right)=0$. For $x \in U$, we have

$$
x=\frac{\left(x, e_{2}\right)}{\left(e_{1}, e_{2}\right)} e_{1}+\frac{\left(x, e_{1}\right)}{\left(e_{1}, e_{2}\right)} e_{2}
$$

Hence, for $x \in V$,

$$
Q\left(x_{U}\right)=2 \frac{\left(x, e_{1}\right)\left(x, e_{2}\right)}{\left(e_{1}, e_{2}\right)}
$$

In the sequel, we shall identify the 1-dimensional subspaces in $V$ with points in $n$-dimensional projective space $P^{n}$. 
Example 1. Let $Q$ have signature $(n, 1)$. The interior of the quadric $Q(x)=0$ in the projective space $P^{n}$ can be identified with $n$-dimensional hyperbolic space $D^{n}$. (This model is called the projective (or Klein) model of hyperbolic space). It is known that the distance $d(x, y)$ between $x, y \in D^{n}$ can be found from

$$
\cosh ^{2} \frac{d(x, y)}{k}=\frac{(x, y)^{2}}{Q(x) Q(y)},
$$

where $k>0$ is a fixed constant. Let $w \in D^{n}$. By (3), $x \in D^{n}$ is $w$-extremal if

$$
(x, w)^{2} \leq(g x, w)^{2}
$$

for all $g \in G$. If $w$ is not a fixed point of $G$, then it follows from (5) that the $w$-reduction region is the Dirichlet polyhedron of $G$ in $D^{n}$ with center at $w$ that is, the set of $x \in D^{n}$ such that $d(x, w) \leq d(x, g w)$ for all $g \in G, g \neq$ id (see e.g. [1]). Thus, by (6), the $w$-reduction region is bounded by the planes $(x, g w-w)=0, g \in G$ (cf. Sec. 2 below).

Example 2. Let $q(z), z=\left(x_{1}, \ldots, x_{n-1}\right)$, be a regular quadratic form with signature $\left(q^{\prime}, p^{\prime}\right)$. Let $V$ be the linear space of Hermitian $2 \times 2$-matrices $x=$ $\left(\begin{array}{cc}x_{0} & z \\ \bar{z} & x_{n}\end{array}\right)$, where the bar denotes conjugation in the Clifford algebra $\mathcal{C}_{n-1}$ (see e.g. [34]). We shall abbreviate $x=\left(x_{0}, z, x_{n}\right) \in V$. Denote

$$
Q(x)=\operatorname{det}(x)=x_{0} x_{n}-z \bar{z}=x_{0} x_{n}-q(z) .
$$

The signature of $Q$ is $\left(p^{\prime}+1, q^{\prime}+1\right)$. The action of the Vahlen group $S V_{n-1}$ (see 34]) on the quadratic space $(V, Q)$ is defined as follows:

$$
x \cdot[g]:=\bar{g}^{t} x g, \quad g \in S V_{n-1} .
$$

We say that $x \in V$ is definite if $q(z)$ is negative definite and $\operatorname{det}(x)=Q(x)>0$. Otherwise, $x$ is indefinite. We denote the sets of definite and indefinite Hermitian matrices in $V$ by $V^{+}$and $V^{-}$respectively.

Let $G$ be a discrete subgroup of $S V_{n-1}$ and let $w=(0,0,1)$. Suppose that $U$ is a 1-dimensional subspace of $V$ spanned by an anisotropic vector $e_{1}$ and let $x=e_{1} \cdot[g]=\left(x_{0}, z, x_{n}\right), g \in G$. Then $(w, x)=x_{0} / 2$ and, by (B),

$$
Q\left(w_{g U}\right)=\frac{x_{0}^{2}}{4 \operatorname{det} x}=\frac{x_{0}^{2}}{4 \operatorname{det} e_{1}} .
$$

Hence

$$
\nu_{o}(U)=\inf \frac{x_{0}^{2}}{4\left|\operatorname{det} e_{1}\right|}
$$

the infimum being taken over all $g \in G$ such that $x_{0} \neq 0$. For certain groups $G$ and integral forms $q$, it is shown in [34] that the spectra of nonzero minima $\mathcal{M}_{o}\left(p^{\prime}, q^{\prime}+1\right)=\mathcal{M}_{o}(1,0), p^{\prime} \neq 0$, and $\mathcal{M}_{o}\left(p^{\prime}+1, q^{\prime}\right)=\mathcal{M}_{o}(0,1), q^{\prime} \neq 0$, with respect to $w$ as well as the $w$-reduction region for 1-dimensional subspaces in $V^{-}$ are discrete. In some cases, explicit results were obtained.

Example 3. Let $q$ in Example 2]be positive definite. The set of definite Hermitian matrices $V^{+}$in the projective space $P^{n}$ can be considered as the projective model $D^{n}$ of $n$-dimensional hyperbolic space, and the set of indefinite Hermitian matrices 
$V^{-}$can be identified with the set of $(n-1)$-dimensional planes in $D^{n}$. Define $\Phi: V^{+} \rightarrow H^{n}$ by

$$
\Phi(x):=\left(\frac{z}{x_{0}}, \frac{\sqrt{\operatorname{det}(x)}}{x_{0}}\right)
$$

$\left(x_{0}>0\right)$. Then $\Phi(x \cdot[g])=g \Phi(x)$ for all $x \in V^{+}$and $g \in S V_{n-1}$ (see 11], p. 250).

The set $V^{+}$consists of subspaces of signature $(1,0)$ in the quadratic space $(V, Q)$. Let $w \in V^{+}$, and let $\mathcal{R}=\mathcal{R}_{w}(1,0)$ be the $w$-reduction region of subspaces in $V^{+}$. It follows from Example 2 that $\Phi(\mathcal{R})$ is the Dirichlet polyhedron with center at $\Phi(w)$ in $H^{n}$ provided $w$ is not a fixed point of $G$.

Let $w=(0,0,1) \in V$. Let $U$ be the subspace of $V$ which is orthogonal to $x=\left(\begin{array}{cc}x_{0} & z \\ \bar{z} & x_{n}\end{array}\right) \in V^{-}, x_{0}>0$. The signature of $U$ is $(n-1,1)$, and $S=\Phi(U)$ is a hemisphere in $H^{n}$ with center at $-z / x_{0}$ and radius $|\operatorname{det}(x)|^{1 / 2} / x_{0}=\left|Q\left(w_{U}\right)\right|^{-1 / 2} / 2$ by (7).

Now let a subspace $U \subset V$ have signature $(1, k), 0<k<n$. We choose an orthogonal basis $e_{1}, \ldots, e_{n-k}$ in $U^{\perp}$ so that $x_{0}\left(e_{1}\right) \neq 0$ and $x_{0}\left(e_{i}\right)=0, i=$ $2, \ldots, n-k$. Let $U_{i}$ be the subspace in $V$ which is orthogonal to $e_{i}, i=1, \ldots, n-$ $k$. Then the $k$-dimensional hemisphere $S=\Phi(U)$ in $H^{n}$ is the intersection of mutually orthogonal vertical planes $\Phi\left(U_{i}\right), i=2, \ldots, n-k$, and the hemisphere $S_{1}=\Phi\left(U_{1}\right)$. Hence the radius of $S$ is equal to $\left|Q\left(w_{U}\right)\right|^{-1 / 2} / 2$, the radius of $S_{1}$. In particular, when $k=1$, and the endpoints of the geodesic $L=\Phi(U)$ are $\eta, \eta^{\prime} \in W$,

$$
\left|Q\left(w_{U}\right)\right|^{-1 / 2}=\left|\eta-\eta^{\prime}\right|
$$

Let $L$ be the axis of a loxodromic element in $G$. Let $k(L)=\sup \left|g(\eta)-g\left(\eta^{\prime}\right)\right|$, the supremum being taken over all $g \in G$. Hence $k^{-2}(L)=\nu(w, U)$. The inequality

$$
|\eta-g(\infty)|<r^{2}(g) / k
$$

holds for infinitely many cosets of $G_{\infty}=\operatorname{Stab}(\infty, G)$ in $G$ when $k=k(L)$, and $k(L)$ is the best constant possible. Here $r(g)$ is the radius of the isometric sphere $I(g)=\left\{x \in H^{n}:\left|g^{\prime}(x)\right|=1\right\}$ of $g \in G$. Thus, the spectrum of $(1,1)$-minima with respect to $w$ is

$$
\mathcal{M}(1,1)=\left\{a^{2}, a \in \mathcal{M}(G)\right\} .
$$

Here $\mathcal{M}(G)$ is the Markov spectrum of $G$ (see [36], 38]).

Let $n>2$. Let $G$ be an arithmetic subgroup of $S V_{n-1}$. For $w=(1,0,0)$, the $w$-reduction region of subspaces of signature $(n-1,1)$ is found in 36 . It is discrete. When $n=3$ and $G$ is a Bianchi group, the region $\mathcal{R}_{w}(2,1)$ is found in 32 and [33]. The elements of $\mathcal{R}_{w}(2,1)$ parametrize the conjugacy classes of maximal Fuchsian subgroups of $G$.

Example 4. Let $Q(x)=a d-b c=\operatorname{det} x$. We identify $V$ with the linear space of $2 \times 2$-matrices $x=\left(\begin{array}{ll}a & b \\ c & d\end{array}\right)$. Let $O_{1}(V)=G L_{2}(\mathbf{R})$ and $G=G L_{2}(\mathbf{Z})$. Let $\mathcal{C}$ be the set of hyperbolic planes $U \in V$ spanned by isotropic vectors $e_{1}=\left(\begin{array}{cc}\theta & 0 \\ 1 & 0\end{array}\right)$ and $e_{2}=\left(\begin{array}{cc}0 & \theta^{\prime} \\ 0 & 1\end{array}\right)$. Then $O_{1}(V) \mathcal{C}=\mathcal{C}$ and $O_{1}(V)$ acts transitively on $\mathcal{C}$. For 
$w=I$, the identity matrix, $g w=g \in G$. By (4),

$$
Q\left(g_{U}\right)=\frac{\left|a-\theta^{\prime} c\right||b-\theta d|}{\left|\theta^{\prime}-\theta\right|} .
$$

In 1947 H. Davenport and H. Heilbronn [10] showed that the first three minima in the spectrum $\mathcal{M}_{w}(1,1)$, which are attained at the $w$-extremal hyperbolic planes with $\left(\theta, \theta^{\prime}\right)=\left(\frac{1-\sqrt{5}}{2}, \frac{1+\sqrt{5}}{2}\right),(\sqrt{2},-\sqrt{2}),(\sqrt{2}, 3-\sqrt{2})$, are equal to

$$
\frac{3-\sqrt{5}}{2 \sqrt{5}}, \quad \frac{2-\sqrt{2}}{4}, \quad \frac{\sqrt{2}-1}{3}
$$

and that the last one is an accumulation point in the spectrum.

If instead of $w=I$, the isotropic vector $u=\left(\begin{array}{ll}1 & 0 \\ 1 & 0\end{array}\right)$ is chosen, then

$$
Q\left((g u)_{U}\right)=\frac{\left|a-\theta^{\prime} c\right||a-\theta c|}{\left|\theta^{\prime}-\theta\right|} .
$$

It follows that $\mathcal{M}_{u}(1,1)$ is the Markov spectrum (see [4]). Thus, only the first two $u$-extremal hyperbolic planes remain extremal upon replacement of $u$ by $w$.

Example 5. Here a Klein model of the hyperbolic plane is introduced. It will be used in Section 7.

Let $n=2$. Let $Q(x)=x_{1}^{2}-4 x_{0} x_{2}$. Then $x \in D^{2}$ if and only if $Q(x)<0 . Q(x)$ is the discriminant of the quadratic form

$$
f_{x}(u)=f_{x}\left(u_{1}, u_{2}\right)=x_{0} u_{1}^{2}+x_{1} u_{1} u_{2}+x_{2} u_{2}^{2}
$$

(cf. [5, p. 301). Two forms $f_{x}$ and $f_{y}$ are said to be equivalent if there is a $g=\left(\begin{array}{ll}a & b \\ c & d\end{array}\right)=\left(\begin{array}{cc}a(g) & b(g) \\ c(g) & d(g)\end{array}\right) \in G L_{2}(\mathbf{Z})$ such that $f_{y}(u)=f_{x}(g u)$. In that case $y=\phi(g)(x)$, where

$$
\phi(g)=\left(\begin{array}{ccc}
a^{2} & a c & c^{2} \\
2 a b & a d+b c & 2 c d \\
b^{2} & b d & d^{2}
\end{array}\right) .
$$

Let $G=\phi\left(G L_{2}(\mathbf{Z})\right) \in O(V)$ and $w=(1,0,0)$. For $g \in G$ with $a \neq 0$ we have $\phi(g) w=\left(a^{2}, 2 a b, b^{2}\right)=a^{2}\left(1,2 z, z^{2}\right)$, where $z=b / a, a, b \in \mathbf{Z}$.

For $x \in P^{2}$, the line $L=\left\{y \in P^{2}:(y, x)=0\right\}$ is said to be the polar of $x$ and $x$ the pole of $L$ (see e.g. [8]). In the model $D^{2}$, indefinite quadratic forms $f_{x}(u)$ are associated with the points $x \in P^{2}$ with $Q(x)>0 . x$ is the pole of the line in $D^{2}$ through the points $e_{k}=\left(1,-2 \theta_{k}, \theta_{k}^{2}\right), k=1,2$, where $f_{x}\left(\theta_{k}, 1\right)=0, k=1,2$, since

$$
\left(x, e_{k}\right)=-2 f_{x}\left(\theta_{k}, 1\right) .
$$

Thus, such a point $x$ (and the form $f_{x}(u)$ ) is associated with the hyperbolic plane $U \subset V$ spanned by $e_{1}$ and $e_{2}$, and by (77) or (8) we get

$$
1 / \nu(U)=\nu_{w}^{2}(x)=\inf \frac{\left|f_{x}(a, b)\right|^{2}}{Q(x)}
$$

the infimum being taken over all $(a, b) \in \mathbf{Z}^{2} /(0,0)$. The following definition is well known (see e.g. [4]): The set of nonnegative numbers $\mathcal{M}=\left\{\nu_{w}(x): x \in\right.$ $\left.P^{2}, Q(x)>0\right\}$ is called the Markov spectrum. 
Definitions and summary of results.

In the sequel, for $g \in G L_{2}(\mathbf{R})$, instead of saying that $\phi(g)$ in (11) acts in $P^{2}$, we say that $g$ acts in $P^{2}$. This should not cause any confusion.

An infinite discrete subgroup of the group of isometries of $D^{2}$ whose limit set contains more than two points is said to be a non-elementary Fuchsian group. Let $H$ be such a group. Let $F_{2}, F_{1} \in H$ be hyperbolic and let $G^{\prime}$ be the subgroup of $H$ generated by $F_{2}$ and $F_{1}$. Assume that there is an involution $S$ such that $F_{2}=S_{1} S$ and $F_{2}=S S_{2}$, where $S_{1}$ and $S_{2}$ are involutions. Let $G$ be the group generated by the involutions $S_{1}, S, S_{2}$. Then $\left[G: G^{\prime}\right]=2$. (Note that the involutions $S_{1}, S$ and $S_{2}$ do not necessarily belong to $H$ ).

Let $T=S_{1} S S_{2}$. Define the tree $\Psi(T)$ of triples of involutions generated by $\left(S_{1}, S, S_{2}\right)$ as follows. Let $S^{\prime}=S_{1} S S_{1}$ and $S^{\prime \prime}=S_{2} S S_{2}$. Then $\left(S^{\prime}, S_{1}, S_{2}\right)$, $\left(S_{1}, S_{2}, S^{\prime \prime}\right) \in \Psi(T)$. Similarly, if $\left(U_{1}, U, U_{2}\right) \in \Psi(T)$ then $\left(U^{\prime}, U_{1}, U_{2}\right),\left(U_{1}, U_{2}, U^{\prime \prime}\right)$ $\in \Psi(T)$. Here $U^{\prime}=U_{1} U U_{1}, U^{\prime \prime}=U_{2} U U_{2}$. By associating to every triple $\left(U_{1}, U, U_{2}\right) \in \Psi(T)$ the indefinite fixed point $f$ of $F=U_{1} U_{2}$ in $P^{2}$ we obtain the tree of indefinite points $\mathcal{F}(T)$ associated with $\Psi(T)$. (We say that a point $x \in P^{2}$ is definite, isotropic or indefinite if $Q(x)<0,=0$ or $>0$ respectively.) Denote by $C_{T}$ the conic in $P^{2}$ with equation $(T x+x, x)=0$. The limit set of the tree $\mathcal{F}(T)$ lies on $C_{T}$ (Theorem 17).

Let $w \in P^{2}$ and let $G$ be a subgroup of a Fuchsian group $H$. Let $D_{T} \subset P^{2}$ be the closed region bounded by $C_{T}$ and the axes of the involutions of $S_{1}$ and $S_{2}$ (see Fig. 6, below). We shall say that

(1) the tree $\mathcal{F}(T)$ is $w$-extremal if every $f \in \mathcal{F}(T)$ is $w$-extremal (that is $(f, g w)^{2}$ $\geq(f, w)^{2}$ for any $\left.g \in H\right)$;

(2) $\mathcal{F}(T)$ is unique in $D_{T}$ if an extremal $f \in D_{T}$ implies $f \in \mathcal{F}(T)$;

(3) $\mathcal{F}(T)$ is simple on $M=D^{2} / G$ if the projection of the polar of any $f \in \mathcal{F}(T)$ is a simple closed geodesic on the Riemann surface $M$.

Denote $A^{2}=\left\{x \in P^{2}:(x, w) \neq 0\right\}$. The points in $A^{2}$ can be normalized by the condition $(x, w)=1$. Let $t$ be the fixed point of $T$. When $w=t$, the equation of $C_{T}$ in $A^{2}$ is $Q(x)=$ const.

Let $H=G$ and $t=w$. In [12, the case when $T$ is elliptic or parabolic was studied and a complete description of the discrete part of $\mathcal{M}(G)$ was obtained. In [23], these results were obtained only for parabolic $T$. In [15], the problem was solved for any $T$ and the spectrum was identified with the set of simple closed geodesics on $M$.

In [26, the discrete part of the spectrum was found when $H$ is the Hecke group $G_{5}, T$ is hyperbolic and $w \neq t$. It was shown that all the points in the spectrum correspond to simple closed geodesics on some Riemann surface; however, there are other simple closed geodesics on the surface.

The main purpose of this paper is to study the $w$-reduction regions for $(0,1)$ and $(1,0)$-subspaces in $(V, Q)$ for a regular quadratic form $Q$ with signature $(2,1)$. Let $G$ be a discrete subgroup of $O(V)$. If $w \in D^{2}$ or $w$ is a parabolic fixed point of $G$, then $\mathcal{R}_{w}(0,1)=G_{w} D(w) \subset D^{2}$, where $D(w)$ is the Dirichlet polygon of $G$ with center at $w$. The sets $\mathcal{R}_{w}(0,1)$ and $\mathcal{R}_{w}(1,0)$ both belong to the projective plane $P^{2}$, and we set $\mathcal{R}_{w}=\mathcal{R}_{w}(0,1) \cup \mathcal{R}_{w}(1,0)$. The method for completely describing $\mathcal{R}_{w}$ inside $D_{T}$ developed in this paper is applicable to many Fuchsian groups. Here it is applied to the groups generated by three involutions (Theorem 21) and to some maximal subgroups of $G L_{2}(\mathbf{R})$ commensurable with the modular group (Examples [30] and [31). In [39], the method is applied to the Hecke groups. 
In Section 3, we prove general criteria for $w$-extremality of an indefinite fixed point of a hyperbolic element in a Fuchsian group. In Sections 4 and 5, they are applied to show that, under a very mild restriction on the position of $w$, if $f_{1}$ and $f_{2}$, the indefinite fixed points of $F_{1}$ and $F_{2}$, are $w$-extremal, then the whole tree $\mathcal{F}(T)$ is $w$-extremal (Theorem 18).

In Section 5, we first consider the case when $H=G$ and $w=t$, and, for any $T$, we prove that the tree $\mathcal{F}(T)$ is unique in the region $D_{T}$ (Theorem 21). Here our approach is close to that of [4]. Applications of this result are given in Examples 25 and 26. In Example 25, it is applied to the Fuchsian group which is the stabilizer of the line $\omega \mathbf{R} \subset \mathbf{C}, \omega=(1+\sqrt{-19}) / 2$, in the Bianchi group $B_{19}=P G L_{2}\left(\mathcal{O}_{19}\right)$, where $\mathcal{O}_{19}$ is the ring of integers of the field $\mathbf{Q}(\sqrt{-19})$. It turns out that the discrete part of the Markov spectrum of this Fuchsian group lies above $1 / \sqrt{5}$, and it can be described in terms of the positive integer solutions of the Diophintine equation $x_{1}^{2}+x_{2}^{2}+5 x^{2}=5 x_{2} x_{1} x$. It follows that $1 / \sqrt{5}$ is a limit point of the Markov spectrum of $B_{19}$. In Example 26, Theorem 21 is applied to the compact triangle groups $(2, q, m), q=3$ or 4 . Then, for an isotropic $w \neq t$, we prove Theorem 29] which can be used to solve the problem of uniqueness of the tree $\mathcal{F}(T)$ in $D_{T}$ for general zonal Fuchsian groups. In particular, it is shown in Section 7 how it can be applied to maximal Fuchsian groups $G(m)$ commensurable with the modular group. The Markov spectra for these groups coincide with the Markov spectra $\mathcal{M}_{m}$ on the sublattices of index $m$, and they are subsets of the classical spectrum $\mathcal{M}=\mathcal{M}_{1}$. For $m=2,5,6$, the only cases, except $m=1$, when $T$ is parabolic, the discrete part of $\mathcal{M}_{m}$ was first found in [27, 29], 30] respectively, and for $m=3$, in 24]. In Example 30 we consider the case $m=3$. In that case, the group $G=G(3)$ is conjugate to the Hecke group $G_{6}$ and, as it is shown in [39], there are two trees $\mathcal{F}\left(T_{2}\right)$ and $\mathcal{F}\left(T_{3}\right)$ of extremal indefinite points, both of them are simple on $D^{2} / G_{S}$, and $\mathcal{F}\left(T_{3}\right)$ is unique in $D_{T_{3}}$. Here $G_{S}$ is the subgroup of $G$ generated by its involutions.

In Example 31, where $G=G(13)$, we find the extremal tree $\mathcal{F}(T)$, show that it is unique inside $C_{T}$, and give a complete description of the discrete part of the Markov spectrum on the sublattice of index 13 (Theorem 32). It leads to the description of a subset of the classical Markov spectrum $\mathcal{M}$ whose limit set is a Cantor set in the interval [0.303983697, 0.303986571]. Note that this subset belongs to the interval $\left(12^{-1 / 2}, 1 / 3\right)$, where not too much is known about the structure of the spectrum $\mathcal{M}$ (see 9 ]).

In Section 8, a simple proof of the simplicity of the tree $\mathcal{F}(T)$ for the group $G$ generated by three involutions is given. This result was first obtained in [13] and [18].

In [39, the results of this paper are applied to the Fuchsian group generated by the reflections in the sides of a right triangle. The intersection of this group with $S L_{2}(\mathbf{R})$ is generated by two elements $S$ and $A$ such that $S^{2}=A^{q}=$ id. We choose $w$ to be the fixed point of $A S$ in $P^{2}$ (for $q=3$ or 4 , see Example 26 below). For this group we find $[(q-1) / 2]$ distinct trees of extremal indefinite points and show that all of them are simple on the Reimann surface $M=D^{2} / G_{S}$, where $G_{S}$ is the group generated by the involutions in $G_{q}$. For the Hecke groups $G_{q}, q \geq 5$, we show the uniqueness of one of the trees, which leads to the complete description of the discrete part of the Markov spectrum for $G_{q}$. In particular, for even $q$, the first accumulation point in the spectrum is $(\cos (\pi / q)) / 2$. For $q=5$, the spectrum was found in [26]. 
The author thanks the referee for useful suggestions which led to an improvement of this work.

\section{2. $g$-STRIPS AND THEIR PROPERTIES}

Let the signature of $Q(x), x \in P^{2}$, be $(2,1)$. A point $x$ belongs to $D^{2}$ if and only if $Q(x)<0$. Denote

$$
C=\left\{x \in P^{2}: Q(x)=0\right\} .
$$

Thus, a point $x \in P^{2}$ is definite, isotropic, or indefinite if $x \in D^{2}, x \in C$ or $x \notin \bar{D}^{2}$ respectively. A hyperbolic $F \in G$ has three fixed points in $P^{2}$; two of them are isotropic and one, $f$, indefinite. In the sequel, we shall sometimes refer to $f$ as the fixed point of $F$ in $P^{2}$. The $w$-minimum of $x \in P^{2}$ will be denoted by $\nu(x)$ instead of $\nu(U)$ as in (1).

Let $w \in P^{2}$ be fixed. For every $g \in G / G_{w}$, we define the $g$-strip to be the set $\left\{x \in P^{2}:(x, g w)^{2}<(x, w)^{2}\right\}$. The $g$-strip will be denoted by $p(w, g)$ or simply by $p(g)$. If $h \in G_{w}$, then the $g h$-strip coincides with the $g$-strip. If $x$ belongs to a $g$-strip, then $x$ is not $w$-extremal and, by definition,

$$
\mathcal{R}_{w}=\mathcal{R}_{w}(0,1) \cup \mathcal{R}_{w}(1,0)=P^{2}-\bigcup p(w, g)
$$

the union being taken over all $g \in G / G_{w}$. Denote by $D^{*}(w)$ the connected open component of $\mathcal{R}_{w}$ with the fixed points of parabolic and boundary hyperbolic elements of $G$ in $P^{2}$ adjoined (see 2, p. 265), and by $D^{*}$ the convex hull in $P^{2}$ of all limit points of $G$. It is clear that $D^{*}(w) \subset D^{*}$, and that $D^{*}(w)=\mathcal{R}_{w}(0,1)$ if $G$ is a Fuchsian group of the first kind.

The boundary of a $g$-strip consists of two lines $L^{+}(g)$ and $L^{-}(g)$ with equations

$$
(x, g w-w)=0 \quad \text { and } \quad(x, g w+w)=0
$$

respectively. Since $(g w-w, g w+w)=0$, it follows that the line $M(g)$ through $w$ and $g w$ cuts $D^{2}$, the pole $(g w-w)$ of $L^{+}(g)$ belongs to $L^{-}(g)$, and the pole $(g w+w)$ of $L^{-}(g)$ belongs to $L^{+}(g)$. Thus, the Dirichlet polyhedron for $G$ with center at $w$ is bounded by the lines $L^{+}(g), g \in G$ (see Example 1 ).

In the sequel, we assume that $w \in D^{*}$. Then

$$
(w, g w)^{2} \geq(w, w)^{2}
$$

for all $g \in G$. Since $Q(g w-w) Q(g w+w) / 4=(w, w)^{2}-(w, g w)^{2} \leq 0$, one and only one of the lines $L^{-}(g)$ and $L^{+}(g)$ cuts $D^{2}$. The pole of $M(g)$ is the point of intersection of the lines $L^{-}(g)$ and $L^{+}(g)$.

Lemma 6. Let $x \in P^{2}$ be the fixed point of $g \in G$ corresponding to the eigenvalue 1 or -1 . If $g x=x$ then $x \in L^{+}(g)$, and if $g x=-x$ then $x \in L^{-}(g)$.

If $g \in G$ is an involution, then $g w+w \in D^{2}$ is the fixed point of $g$, and the boundary $L^{-}(g)$ of the $g$-strip is invariant with respect to the position of $w$.

If $g \in G$ is a reflection, then $g w-w \notin D^{2}$ is the fixed point of $g$, and the boundary $L^{+}(g)$ of the $g$-strip is the axis of $g$; hence it is invariant with respect to the position of $w$.

Proof. If $g^{-1} x=x$ then $\left(g^{-1} x, w\right)=(x, g w)=(x, w)$ and $x \in L^{+}(g)$, and if $g^{-1} x=-x$ then $\left(g^{-1} x, w\right)=(x, g w)=-(x, w)$ and $x \in L^{-}(g)$.

If $g$ is an involution or reflection, then $g(g w+w)=w+g w$, and $g(g w-w)=$ $-(g w-w)$. 
The following result can also be useful.

Corollary 7. Let $s$ and $t$ be the fixed points of $S, T \in G$, where $S$ is an involution. If $s, t$ and $w$ are collinear, then the $S$ - and $S T$-strips coincide.

Proof. If $w=t$ then the $S$ - and $S T$-strips coincide. Denote by $M$ the line through $s$ and $t$ and by $p_{M}$ the pole of $M$. Then $p_{M}$ does not depend of the position of $w$ on $M$. Let $f$ be the fixed point of $S T$. By Lemma 6] $f, p_{M} \in L^{+}(S T)$ for any $w \in M$. Thus, $L^{+}(S T)=L^{+}(S)$ for $w \in M$. By Lemma [6] $u$, the pole of $L^{+}(S)$, lies on $L^{-}(S T)$. Since $p_{M} \in L^{-}(S T), L^{-}(S T)=L^{-}(S)$ for any $w \in M$, as required.

Recall that $A^{2}=\left\{x \in P^{2}:(x, w) \neq 0\right\}$. We shall normalize $x \in A^{2}$ by the condition

$$
(x, w)=1 .
$$

Then a point $x \in A^{2}$ is $w$-extremal if and only if $(x, g w)^{2} \geq 1$ for all $g \in G$.

Let $R$ be a region in $A^{2}$. Assume that for any $w$-extremal point $x \in R$ the following dichotomy occurs: either $(x, g w) \leq-1$, or $(x, h w) \geq 1$ for some $g, h \in G$. This means there are $g=g_{0}, g_{1}, \ldots, g_{k-1}, g_{k}=h \in G$ such that if $(x, g w)>-1$ and $(x, h w)<1$, then $x \in R$ is not $w$-extremal and $\left(x, g_{i} w\right)^{2}<1$ for at least

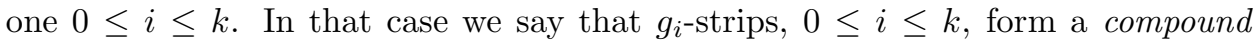
$(g, h)$-strip (or simply a $(g, h)$-strip) in $R$.

If $x \in P^{2}$ is $w$-extremal, i.e. $(x, g w)^{2} \geq(x, w)^{2}$ for all $g \in G$, then $\left(w, g^{-1} x\right)^{2} \geq$ $(w, x)^{2}$ for all $g \in G$, i.e. $w$ is $x$-extremal. In the sequel, this statement will be called the duality principle.

\section{Extremality CRITERIA}

In this paper, we are mainly concerned with finding the $w$-reduction regions for maximal discrete groups $G$. This is partially justified by the simple fact that the $w$-extremality of $f \in P^{2}$ with respect to $G$ implies the $w$-extremality of $f$ with respect to any subgroup of $G$.

Let $g \in G$. The boundary of $p(g)$ consists of two lines $(x, g w \pm w)=0$. Since $g^{-1}(g w \pm w)= \pm\left(g^{-1} w \pm w\right), g^{-1} p(g)$ is the exterior of the strip $p\left(g^{-1}\right)$ and vice versa. Thus,

$$
g^{-1} M(g)=M\left(g^{-1}\right), \quad g^{-1} L^{+}(g)=L^{+}\left(g^{-1}\right), \quad g^{-1} L^{-}(g)=L^{-}\left(g^{-1}\right) .
$$

Let $x \in A^{2}$. Assume that $G_{x}$, the stabilizer of $x$ in $G$, is a cyclic group generated by $h \in G$. It follows that the fundamental domain of $G_{x}$ is bounded by the lines $L^{+}(h)$ and $L^{+}\left(h^{-1}\right)$, which meet at $x$.

The following simple criterion of extremality is proved in [36] (see also [38]).

Lemma 8. Let $x \in A^{2}$ be the pole of a line $L$. Then $x$ is $w$-extremal if the fundamental domain of $G_{x}$ on $L$ belongs to $D^{*}(w)$, the connected component of $\mathcal{R}_{w}$.

Denote by $K_{x}$ the intersection of a fundamental domain of $G_{x}$ in $D^{*}$ with the orbit $G w$. The following statement is evident.

Lemma 9. Suppose that the points $f_{1}, \ldots, f_{r} \in A^{2}$ are $w$-extremal. For $f \in A^{2}$, denote

$$
R_{i}=\left\{x \in A^{2}:(f, x)^{2} \geq\left(f_{i}, x\right)^{2}\right\}, \quad i=1, \ldots, r .
$$

Let $D^{\prime \prime}$ be a fundamental domain of $G$ in $D^{*}$ such that $w \in D^{\prime \prime}$. Let $D^{\prime}=G_{w} D^{\prime \prime}$. If $K_{f} \subset R_{1} \cup \cdots \cup R_{r} \cup D^{\prime}$, then $f$ is $w$-extremal. 


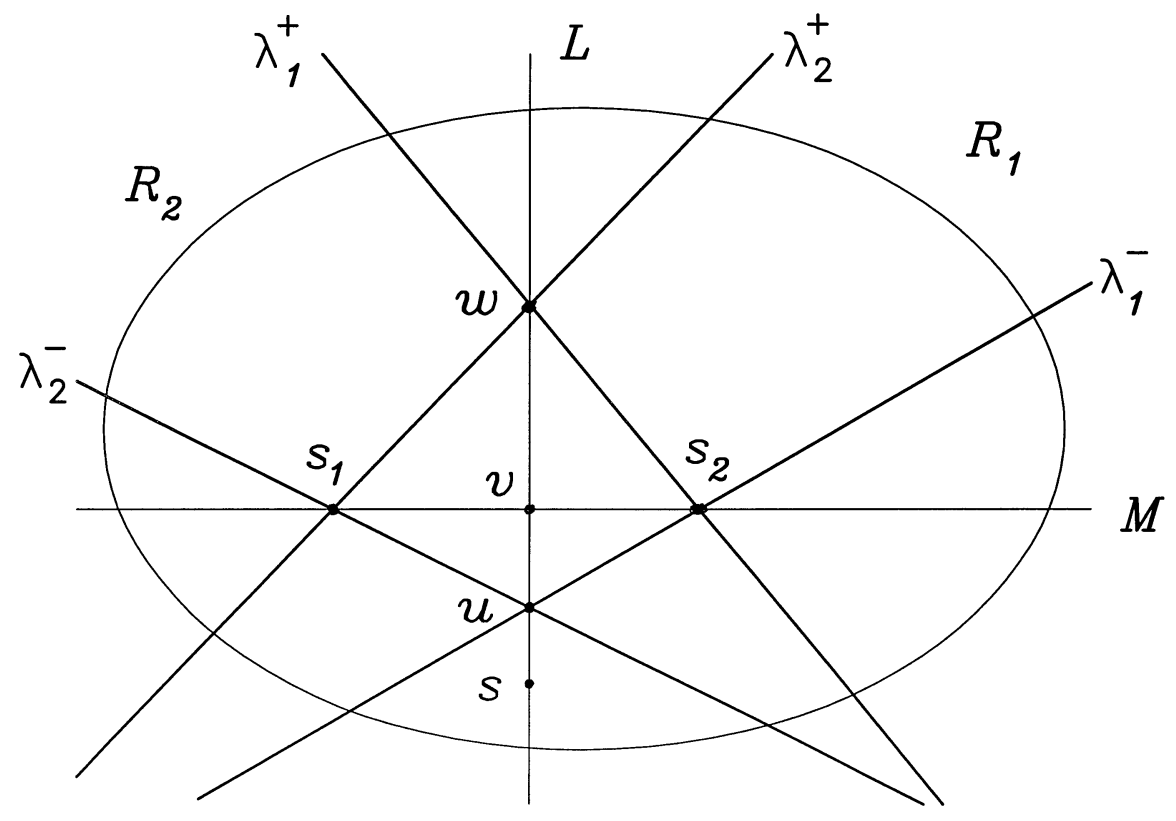

FiguRE 1.

Let $w$-extremal indefinite points $f_{1}, f_{2} \in A^{2}$ be the fixed points of hyperbolic $F_{1}, F_{2} \in G$. Assume that the line $L_{3}$ through $f_{1}$ and $f_{2}$ does not cut $C$. Let $S \in O(V)$ be the involution whose fixed point $s \in D^{2}$ is the pole of $L_{3}$. Note that $S$ fixes $L_{3}$ pointwise and that $S x=-x$ for every $x \in L_{3}$. There are unique involutions $S_{1}, S_{2} \in O(V)$ such that

$$
F_{1}=S S_{2}, \quad F_{2}=S_{1} S .
$$

Denote by $s_{k} \in D^{2}$ the fixed point $S_{k}$ and by $L_{k}$ the polar of $s_{k}, k=1,2$. Let

$$
F=F_{2} F_{1}=S_{1} S_{2}
$$

and let $f \in A^{2}$ be the indefinite fixed point of $F$. Note that if $S_{k} \in G$ then $L_{k}=L^{-}\left(S_{k}\right)$. The region $R_{k} \subset A^{2}$ in (14) is bounded by two lines:

$$
\lambda_{k}^{ \pm}=\left\{x \in A^{2}:(f, x)= \pm\left(f_{k}, x\right)\right\}, \quad k=1,2,
$$

which meet at $s_{j}, k+j=3$. The lines $\lambda_{1}^{+}$and $\lambda_{2}^{+}$meet at $w$. Denote $u=\lambda_{1}^{-} \cap \lambda_{2}^{-}$. Then the points $w, u$, and $s$ lie on the line

$$
L=\left\{x \in A^{2}:\left(f_{1}, x\right)=\left(f_{2}, x\right)\right\}
$$

(see Fig. 1). Let $J$ be the involution in $P^{2}$ determined by the point $s$ and the line $M$, the axis of $F$. Let $v$ be the point of intersection of the line through $u$ and $w$ with $M$. Let $u=\lambda s+v$ and $w=\mu s+v$. Then $(f, u)=\lambda(f, s)=-1,(f, w)=\lambda(f, s)=1$. Hence $\lambda=-\mu, J(u)=w$, and $u$ is located on $L \cap D^{*}$ between $s$ and $v$.

Lemma 10. Suppose that $w \in D^{*}$ is either 1) an indefinite point and $S \in G$, or 2) an isotropic point in $P^{2}$. With the notation introduced above, assume that the quadrilateral with vertices at $w, s_{1}, s$, and $s_{2}$ is convex. Then the indefinite point $f$ is w-extremal. 


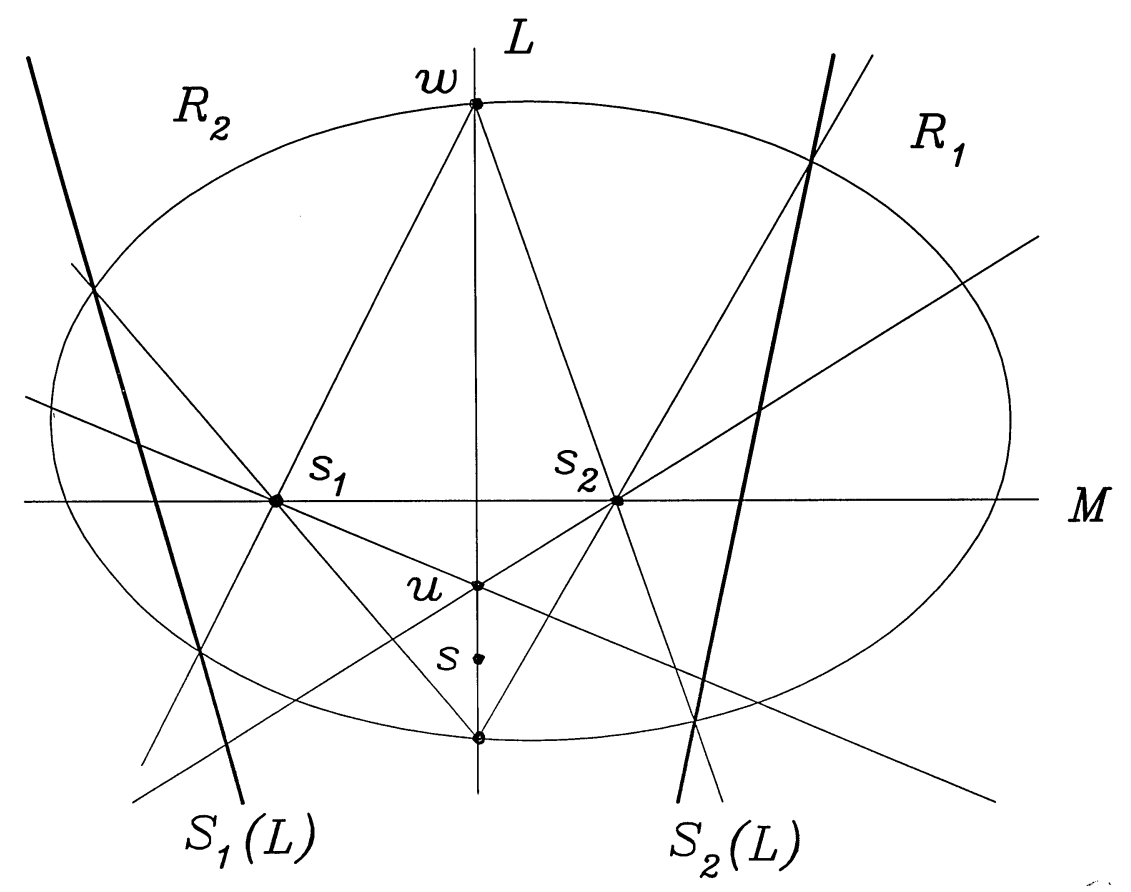

FiguRE 2.

Proof. It is clear that the region in $D^{*}$ bounded by the lines $S_{1}(L)$ and $S_{2}(L)$, where $L$ is defined by (15), is a fundamental domain $\Phi$ of the cyclic group generated by $F \in G$. Denote the parts of $\Phi$ between the lines $L$ and $S_{1}(L)$ and between $L$ and $S_{2}(L)$ by $\Phi_{2}$ and $\Phi_{1}$ respectively.

Assume first that $w$ is an isotropic point. Then $G w \subset C$. Since $u \in D^{2}$, $G w \cap \Phi_{k} \subset R_{k}, k=1,2$. (In Figure 2, two lines through $w$ and $s_{2}$ and through $S(w)$ and $s_{2}$ are used to construct the line $S_{2}(L)$. As in Figure 1, the region $R_{1}$ is bounded by the lines through $w$ and $s_{2}$ and through $u$ and $s_{2}$.) Thus, by Lemma $9 f$ is $w$-extremal.

Now let $w$ be an indefinite point. Then the triangle with vertices at $w, s_{1}$, and $s_{2}$ does not contain any $g w$, id $\neq g \in G$. As above, it follows that $f$ is $w$-extremal.

\section{Three elliptic elements of ORDER two}

Let $G$ be a group generated by three involutions $S_{1}, S_{2}, S$ fixing the points $s_{1}, s_{2}, s$ in $D^{2}$, which are not collinear. In this section, we abbreviate $\operatorname{tr}(F)=$ $\operatorname{tr}\left(\phi^{-1} F\right)$, where $\phi: H^{2} \rightarrow D^{2}$ is an isometry, so that $\phi^{-1}(G) \subset S L_{2}(\mathbf{R})$ (see e.g. (11)). Let

$$
F_{1}=S S_{2}, F_{2}=S_{1} S, F=S_{1} S_{2}
$$

Then the lengths $a_{1}, a$ and $a_{2}$ of the sides of the triangle with vertices $s_{1}, s$ and $s_{2}$ can be found from

$$
m_{1}=2 \cosh a_{1}=\left|\operatorname{tr}\left(F_{1}\right)\right|, \quad m_{2}=2 \cosh a_{2}=\left|\operatorname{tr}\left(F_{2}\right)\right|, \quad m=2 \cosh a=|\operatorname{tr}(F)| .
$$

Let $\alpha_{1}, \alpha, \alpha_{2}$ be the angles of the triangle (see Fig. 3). Let

$$
T=S_{1} S S_{2} .
$$




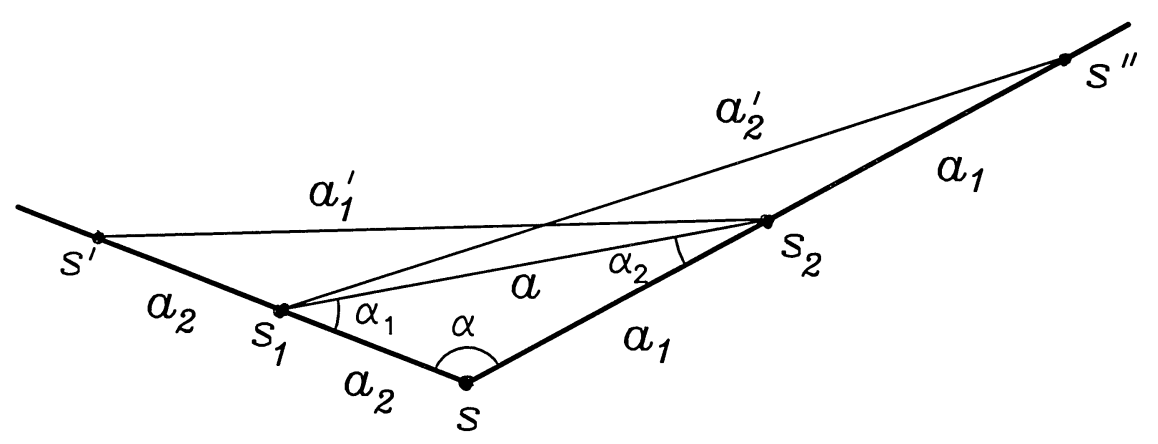

FIGURE 3.

Then

$$
|\operatorname{tr}(T)|=2 \lambda=\sinh a_{1} \sinh a_{2} \sin \alpha,
$$

and $T$ is elliptic, parabolic or hyperbolic according as $\lambda<1, \lambda=1$ or $\lambda>1$ (see [2] p. 301). Positive numbers $m_{1}, m$ and $m_{2}$ satisfy the following "Diophantine" equation (see [2], p. 14):

$$
m_{1}^{2}+m^{2}+m_{2}^{2}=m_{1} m m_{2}+4\left(1-\lambda^{2}\right) .
$$

Assume that $m_{1}<m_{2}<m$. Then the solution $\left(m_{1}, m, m_{2}\right)$ of (17) gives rise to three distinct solutions

$$
\left(m_{1}^{\prime}, m, m_{2}\right), \quad\left(m_{1}, m, m_{2}^{\prime}\right), \quad\left(m_{1}, m^{\prime}, m_{2}\right)
$$

where, by the Cosine Rule I (see [2], p. 148),

$$
m_{1}^{\prime}+m_{1}=m m_{2}, \quad m_{2}^{\prime}+m_{2}=m m_{1}, \quad m^{\prime}+m=m_{1} m_{2} .
$$

(For example, the first formula follows from $m_{1}=m m_{2}-\sinh a \sinh a_{2} \cos \alpha_{1}$ and $m_{1}^{\prime}=m m_{2}+\sinh a \sinh a_{2} \cos \alpha_{1}$ (see Fig. 3)). Denote

$$
S^{\prime}=S_{1} S S_{1}, \quad S^{\prime \prime}=S_{2} S S_{2}, \quad S_{1}^{\prime}=S S_{1} S .
$$

The associated triples of involutions are

$$
\left(S^{\prime}, S_{1}, S_{2}\right), \quad\left(S_{1}, S_{2}, S^{\prime \prime}\right), \quad\left(S, S_{1}^{\prime}, S_{2}\right),
$$

each of which satisfies (16). We shall say that these three triples of involutions are neighbors of the original one. If $\alpha>\pi / 2$, then $\alpha_{1}<\pi / 2, \alpha_{2}<\pi / 2$. Hence

$$
m^{\prime}<m<\min \left(m_{1}^{\prime}, m_{2}^{\prime}\right) \text {. }
$$

We define the height of the solution $\left(m_{1}, m, m_{2}\right)$ (and of the triple $\left(S_{1}, S, S_{2}\right)$, and of the triangle in $P^{2}$ with vertices $\left.s_{1}, s, s_{2}\right)$ to be the positive number $h t\left(m_{1}, m, m_{2}\right)=$ $m_{1}+m+m_{2}$. By (19), if the triangle with vertices $s_{1}, s$ and $s_{2}$ is obtuse then two of its neighbors have larger heights, one has a smaller height, and the triangles with larger heights both are obtuse. Thus there is a solution (and the triple of involutions) of the smallest height, which is said to be singular. If $\left(S_{1}, S, S_{2}\right)$ is singular, then the triangle with vertices $s_{1}, s$ and $s_{2}$ is either acute or right (cf. [25]). 


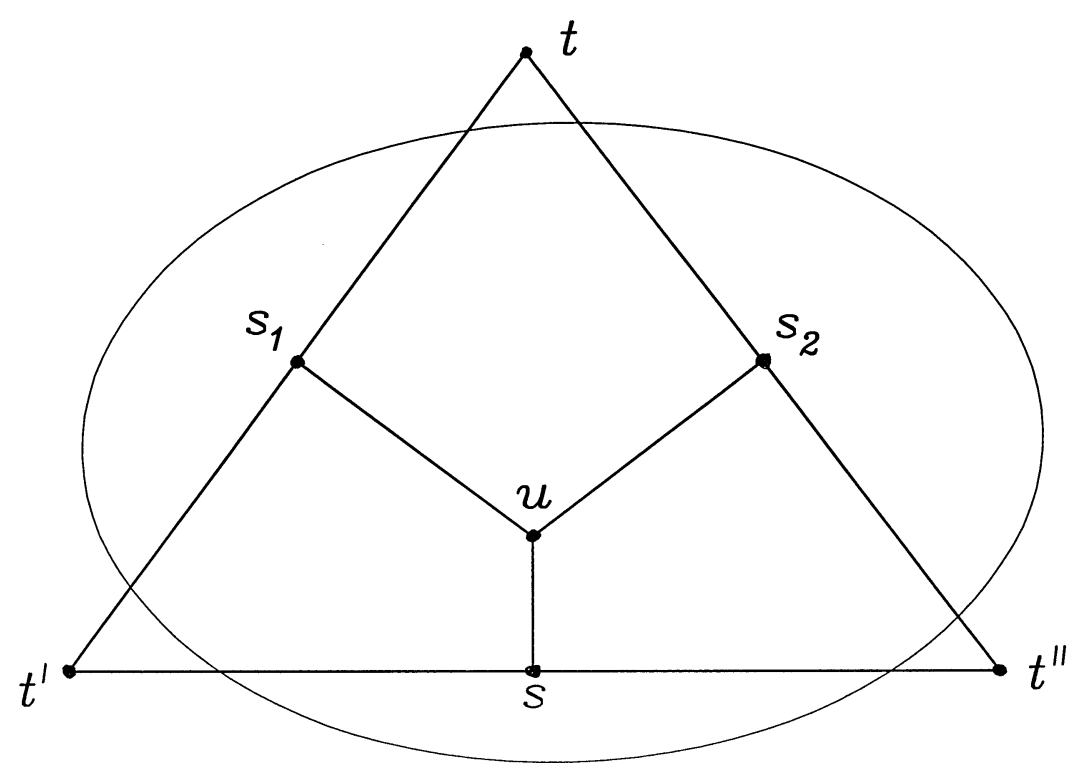

FIGURE 4.

Lemma 11. Let $G$ be the group generated by involutions $S_{1}, S_{2}$ and $S$. Let $T=$ $S_{1} S S_{2}$ and let $t$ be the fixed point of $T$ in $P^{2}$. Assume that $w$ belongs to the triangle with vertices $t, s_{1}$, and $s_{2}$. If the fixed points $f_{1}$ and $f_{2}$ of $F_{1}$ and $F_{2}$ are w-extremal, then the indefinite fixed point $f$ of $F_{2} F_{1}$ is also w-extremal.

Proof. Let $t \in A^{2}$ be the fixed point of $T$. Denote $t^{\prime}=S_{1} t, t^{\prime \prime}=S t^{\prime}$. Then $t=S_{2} t^{\prime \prime}$, and the triangle $\Delta$ with vertices $t, t^{\prime}$, and $t^{\prime \prime}$ is a fundamental domain of $G$ (see Fig. 4 ). The triangle $\Delta$ covers the convex quadrilateral with vertices at $w, s_{1}, s$, and $s_{2}$. Hence the union of $R_{1}, R_{2}$, and $\Delta$ cover $K(f)$, and by Lemma 9 $f$ is $w$-extremal.

Lemma 12. The common perpendicular to the axis of $F$ and the line $L$ through $t^{\prime}$ and $t^{\prime \prime}$ passes through $s$.

Proof. We have to show that the points $f, s$, and $d$, the pole of $L$, are collinear. It is clear that $s$ and $d$ lie on the line $\left(x, t^{\prime}\right)=\left(x, t^{\prime \prime}\right)$. By definition, $t^{\prime}=S T S t^{\prime}=$ $S_{1} S_{2} S t^{\prime}=S_{1} S_{2} t^{\prime \prime}$. Hence $\left(f, t^{\prime}\right)=\left(f, t^{\prime \prime}\right)$, as required.

Corollary 13. The points $t, w$ and $s_{1}$ are collinear in $P^{2}$ if and only if $\left(f_{1}, S_{1} w\right)=$ $\left(f_{1}, w\right)$ (that is $f_{1} \in L^{+}\left(S_{1}\right)$ ).

Proof. By Lemma 12 the line $L^{+}\left(S_{1}\right)$ is orthogonal to both the polar of $f_{1}$ and the line through $t$ and $s_{1}$. But it is also perpendicular to the line through $w$ and $s_{1}=S_{1} w+w$ (see Sec. 2). Hence $t, w$ and $s_{1}$ are collinear.

Let $u \in D^{2}$ be the point of intersection of the heights of the triangle with vertices $s_{1}, s$ and $s_{2}$ (see Fig. 4). By Lemma $12(u, t)=\left(u, t^{\prime}\right)=\left(u, t^{\prime \prime}\right)$. Let $w=u$. Then $\left(S u-u, t^{\prime}\right)=\left(u, t^{\prime \prime}\right)-\left(u, t^{\prime}\right)=0$. Hence $d=S u-u$ and $t^{\prime}, t^{\prime \prime}, s \in L^{+}(S)$. Similarly, $t^{\prime}, t, s_{1} \in L^{+}\left(S_{1}\right)$ and $t^{\prime \prime}, t, s_{2} \in L^{+}\left(S_{2}\right)$. Assume that the triangle with vertices $s_{1}, s$ and $s_{2}$ is singular. By Lemma 12, if the triangle is acute then $u$ is an 


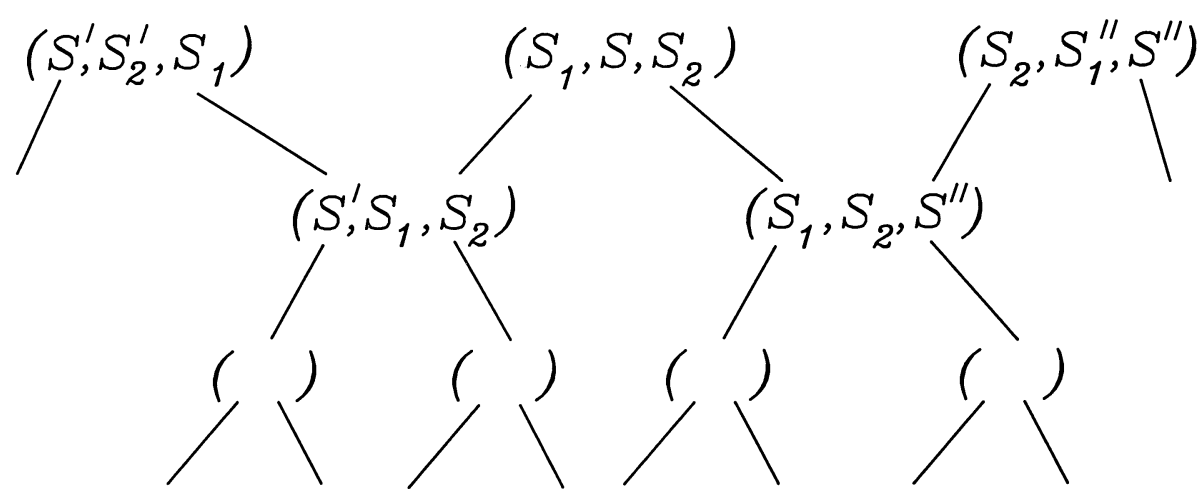

FIGURE 5.

interior point of the triangle, and if it is right and $\alpha=\pi / 2$ then $u=s$. Thus we have obtained the following.

Lemma 14. Let the triangle with vertices $s_{1}, s$ and $s_{2}$ be singular. Let $u \in D^{2}$ be the point of intersection of its heights. If the triangle is acute, then $D^{*}(u)=\Delta$. If it is right and $\alpha=\pi / 2$, then $D^{*}(u)=\Delta \cup S \Delta$.

Corollary 15. The vertices of $D^{*}(t)$ are the points $u, u^{\prime}=S_{1}(u), u^{\prime \prime}=S_{2}(u)$ and their images under the action of the cyclic group $\langle T\rangle$.

Proof. By Lemma $14 t$ is $u$-extremal. The duality principle implies that $u$ is $t$ extremal.

Let the involutions $S^{\prime}$ and $S^{\prime \prime}$ be defined by (18). Let $S_{2}^{\prime}=S_{1} S_{2} S_{1}$ and $S_{1}^{\prime \prime}=$ $S_{2} S_{1} S_{2}$. It is easily seen that the triples

$$
\left(S^{\prime}, S_{2}^{\prime}, S_{1}\right), \quad\left(S_{1}, S, S_{2}\right), \quad\left(S_{2}, S_{1}^{\prime \prime}, S^{\prime \prime}\right)
$$

and their conjugates by the elements in $\langle T\rangle$ are singular. Each pair of adjacent singular triples has a common nonsingular neighbor.

Thus a nonsingular triple $\left(S^{\prime}, S_{1}, S_{2}\right)$ is a common neighbor of $\left(S^{\prime}, S_{2}^{\prime}, S_{1}\right)$ and $\left(S_{1}, S, S_{2}\right)$, a nonsingular triple $\left(S_{1}, S_{2}, S^{\prime \prime}\right)$ is a common neighbor of $\left(S_{1}, S, S_{2}\right)$ and $\left(S_{2}, S_{1}^{\prime \prime}, S^{\prime \prime}\right)$, etc. (see Fig. 5).

The points $s^{\prime}, s_{1}, s_{2}, s^{\prime \prime}=T^{-1} s^{\prime}, T^{-1} s_{1}, T^{-1} s_{2}, \ldots$ lie on the boundary of $D^{*}(t)$. By Lemma 8 the fixed points of $F_{1}, F_{2}, F$, as well as of $F_{1}^{\prime}=S^{\prime} S_{2}$ and $F_{2}^{\prime}=S_{1} S^{\prime \prime}$, are $t$-extremal.

Lemma 16. Let $\left(S_{1}, S, S_{2}\right)$ be a singular triple of involutions. Let $w \in \Delta^{\prime}$ where $\Delta^{\prime}$ is the triangle $\Delta$ with intervals $\left[s_{1}, s_{2}\right],\left[s_{2}, s\right]$ and $\left[s, s_{1}\right]$ removed. Then the indefinite points $f, f_{1}$ and $f_{2}$ are w-extremal.

Proof. The region $D^{*}(f)=G_{f} \Delta$, where $G_{f}=\left\langle S_{1}, S_{2}\right\rangle$. Since $\Delta=D^{*}\left(f_{1}\right) \cap D^{*}(f) \cap$ $D^{*}\left(f_{2}\right)$, the lemma follows from the duality principle.

\section{Trees of $w$-eXtremal indefinite points}

As above, let $G$ be the group generated by three involutions $S_{1}, S$, and $S_{2}$, and $T=S_{1} S S_{2}$. Given a triple of involutions $\left(S_{1}, S, S_{2}\right)$, denote

$$
S_{1}^{\prime}=S_{1} S S_{1}, S^{\prime}=S_{1}, S_{2}^{\prime}=S_{2} \quad \text { and } \quad S_{1}^{\prime \prime}=S_{1}, S^{\prime \prime}=S_{2}, S_{2}^{\prime \prime}=S_{2} S S_{2} \text {. }
$$


Then $T=S_{1}^{\prime} S^{\prime} S_{2}^{\prime}=S_{1}^{\prime \prime} S^{\prime \prime} S_{2}^{\prime \prime}$. Two neighbors

$$
\left(S_{1}^{\prime}, S^{\prime}, S_{2}^{\prime}\right) \text { and }\left(S_{1}^{\prime \prime}, S^{\prime \prime}, S_{2}^{\prime \prime}\right)
$$

of $\left(S_{1}, S, S_{2}\right)$ are nonsingular, i.e. their triangles are obtuse, and their heights are larger than the height of $\left(S_{1}, S, S_{2}\right)$. By taking neighbors with larger height successively we obtain an infinite binary tree of triples of involutions $\Psi(T)$. By associating to each $\left(S_{1}, S, S_{2}\right) \in \Psi(T)$ the indefinite fixed point of $F=S_{1} S_{2}$ we obtain the associated tree of indefinite points $\mathcal{F}(T)$.

Assume that $w$ belongs to the intersection of the triangles with vertices at $t, s_{1}, s_{2}$, at $t, s_{1}^{\prime}, s_{2}^{\prime}$, and at $t, s_{1}^{\prime \prime}, s_{2}^{\prime \prime}$. Assume that $f_{1}$ and $f_{2}$ are $w$-extremal. Then by Lemma 11 the indefinite point $f$ as well as the points $f^{\prime}$ and $f^{\prime \prime}$, the fixed points of $S_{1}^{\prime} S_{2}^{\prime}$ and $S_{1}^{\prime \prime} S_{2}^{\prime \prime}$, are $w$-extremal. Denote by $P_{T}$ the intersection of all such triangles taken over all the triples of involutions in $\Psi(T)$. Then all the points in the tree $\mathcal{F}(T)$ are $w$-extremal provided $w \in P_{T}$.

The limit w-extremal indefinite points.

Consider all the triples $\left(U_{n}, U_{n+1}, U\right) \in \Psi(T), n=0,1, \ldots$, with the involution $U$ fixed. Then $U_{n+1}=U_{n} U_{n-1} U_{n}$. Let $y$ be the fixed indefinite point of $Y=$ $U_{0} U_{1}=U_{n} U_{n+1}$. Then $U_{n} y=-y$. Let $\theta \in C$ be the point of tangency of the tangent line $L$ to $C$ through $y$. Let $x_{n} \in \mathcal{F}(T)$ be the fixed indefinite point of $U_{n} U$. Then $U x_{n}=-x_{n}$. Let $x=\lim _{n \rightarrow \infty} x_{n}$. Then $\theta, y, x \in L$. Thus, $x$ is the point of intersection of the tangent lines $L$ and $U(L)=U Y^{-1}(L)=T^{-1}(L)$ to $C$. Hence

$$
(x, \theta)=(T x, \theta)=0 .
$$

Define

$$
C_{T}=\left\{x \in P^{2}:(x, \theta)=(T x, \theta)=(\theta, \theta)=0\right\} .
$$

By (21), the limit points of the tree $\mathcal{F}(T)$ lie on the curve $C_{T}$. We shall derive the equation of $C_{T}$. Let $x \in C_{T}$. From (21), $\theta=a x+b T x$ for some real numbers $a$ and $b$, and

$$
(\theta, x)=a(x, x)+b(T x, x)=0, \quad(\theta, T x)=a(x, T x)+b(x, x)=0 .
$$

It follows that $a^{2}=b^{2}$. Let $t$ be the fixed point of $T$. If $a=-b$, then $(\theta, t)=0$ for any $\theta \in C$, which is impossible. Thus, $a=b$, and $\theta=x+T x$ in $P^{2}$. By (22), $C_{T}$ is the conic with equation

$$
(T x+x, x)=0 .
$$

Let $G$ be the group generated by three involutions $S_{1}, S, S_{2}$. Let $\Psi(T), T=$ $S_{1} S S_{2}$, be the tree of triples of involutions generated by $\left(S_{1}, S, S_{2}\right)$, and let $\mathcal{F}(T)$ be the corresponding tree of indefinite points in $P^{2}$.

Denote by $\Delta_{F}$ the limit set of the tree $\mathcal{F}(T)$. Let $f_{1}$ and $f_{2}$ be the fixed points of $S S_{2}$ and $S_{1} S$. Let $L_{1}$ and $L_{2}$ be the two tangent lines to $C$ through $f_{1}$ and $f_{2}$ respectively which do not meet inside $C_{T}$ (see Fig. 6). Let $u_{i}$ be the point of intersection of $L_{i}$ with $C_{T}(i=1,2)$. Then $\Delta_{F}$ belongs to the $\operatorname{arc}$ of $C_{T}$ with endpoints $u_{1}$ and $u_{2}$. We have obtained the following.

Theorem 17. The limit set $\Delta_{F}$ of the tree $\mathcal{F}(T)$ is the closure

a) of the set of intersection of the axes of all the involutions which appear in the tree $\Psi(T)$ with $C_{T}$; or

b) of the set of intersection of $C_{T}$ with all the tangent lines to the absolute $C$ through all the points in the tree $\mathcal{F}(T)$. 


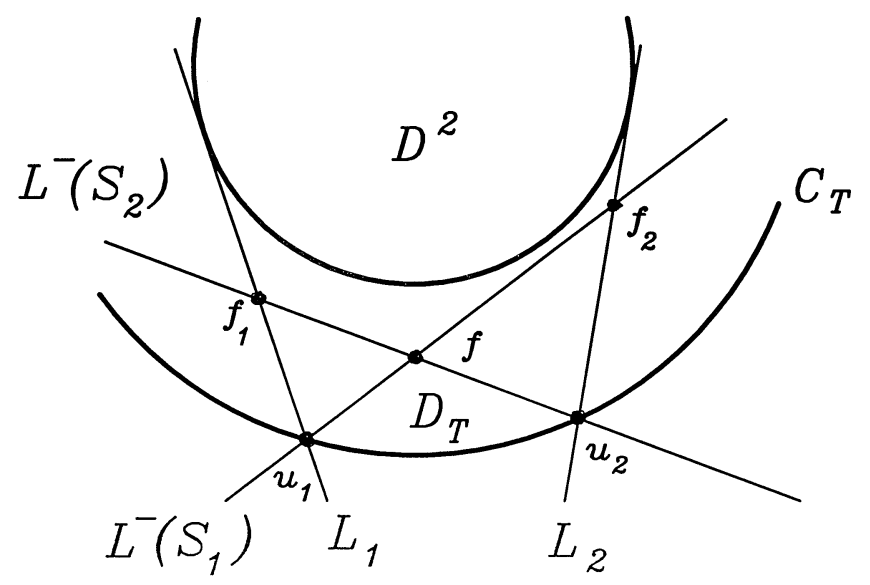

FIGURE 6.

Since $T C_{T}=C_{T}$, the conic $C_{T}$ belongs to the pencil $\mathcal{P}_{T}$ of conics generated by $C$ and $(x, t)^{2}=0$, the polar of the fixed point $t$ of $T$. Thus, the equation of $C_{T}$ can also be written in the form $\nu_{\infty} Q(x)-(x, t)^{2}=0$. Hence, if $w=t$, then by (3) the $w$-minimum of $x \in C_{T}$ equals

$$
\nu(x)=(x, t)^{2} / Q(x)=\nu_{\infty} .
$$

Denote by $C_{T}^{\prime}$ the dual conic for $C_{T}$ in $\mathcal{P}_{T}$. When $T$ is hyperbolic, the hypercycle $C_{T}^{\prime}$ and the point $w$ belong to the different connected components into which the axis of $T$ divides $D^{*}(w)$. (Recall that $C_{T}^{\prime}$ is the envelope of the polars of points of $C_{T}$.) Thus $C_{T}^{\prime} \subset D^{2}$, and $C_{T}^{\prime}$ is a circle with center at $t$, a horocycle with base at $t$, or a hypercycle which has the same end-points as the axis of $T$, if $T$ is elliptic, parabolic, or hyperbolic respectively (see [2], p. 168). Let $B_{T} \subset D^{2}$ be the interior of $C_{T}^{\prime}$ if $T$ is elliptic or parabolic, or the part of $D^{*}(t)$ which is bounded by $C_{T}^{\prime}$ and contains the axis of $T$ if $T$ is hyperbolic. Recall that $\Delta$ is the triangle with vertices $t, S_{1} t, S_{2} t$. Thus, $\Delta \cap \bar{B}_{T} \subset P_{T}$, and, by Lemmas 10 and 11, we have obtained the following.

Theorem 18. Suppose $H$ is a Fuchsian group and $F_{1}, F_{2} \in H$ are hyperbolic. Let $f_{1}$ and $f_{2}$ be the indefinite fixed points of $F_{1}$ and $F_{2}$. Let $S$ be the common involution of $f_{1}$ and $f_{2}$, and let $T=F_{2} S F_{1}$.

Let $w \in \Delta \cap \bar{B}_{T}$. If $w$ is indefinite and $S \in H$, or $w$ is isotropic, or $w$ is definite and $H=\left\langle S_{1}, S, S_{2}\right\rangle$, where $S_{1}=F_{2} S, S_{2}=S F_{1}$, then all the indefinite points in the tree $\mathcal{F}(T)$ are $w$-extremal provided $f_{1}$ and $f_{2}$ are $w$-extremal. All the limit points of $\mathcal{F}(T)$ lie on the conic $C_{T}$ with equation (23).

Lemma [16] and Theorem[18]imply the following.

Corollary 19. Let $G$ be a discrete group generated by three involutions $S_{1}, S, S_{2}$. Let $T=S_{1} S S_{2}, F_{1}=S S_{2}, F_{2}=S_{1} S$. Suppose that the triple $\left(S_{1}, S, S_{2}\right)$ is singular. If $w \in \Delta \cap \bar{B}_{T}$, then $f_{1}$ and $f_{2}$, the fixed points of $F_{1}$ and $F_{2}$, as well as all the points in the tree $\mathcal{F}(T)$, are $w$-extremal.

Remarks. 1. Let $H$ be a Fuchsian group, and let the involutions $S_{1}, S, S_{2} \in H$. Let $\left(S_{1}, S, S_{2}\right) \in \Psi(T)$, where $T=S_{1} S S_{2}$. Let $w$ be definite. It follows from the results 
obtained in $\S 4$ that to prove that the tree $\mathcal{F}(T)$ is $w$-extremal we have to show that $(f, g w)^{2} \geq 1$ for any $g w, g \in H$, in the quadrilateral with vertices at $w, s_{1}, s$ and $s_{2}$. In [39] is done for $H$, a triangle group $(2, q, m)$, and $w$, the fixed elliptic point of order $m$. It is shown there that this quadrilateral does not contain an element of the orbit $H w$ other than $w$ for any $\left(S_{1}, S, S_{2}\right) \in \Psi(T)$.

2. If $w \notin P_{T}$, the structure of the tree of $w$-extremal indefinite points can be destroyed. Let $G=S L_{2}(\mathbf{Z})$. When $w=u, \nu\left(f_{1}\right)=\nu\left(f_{2}\right)$ is the Hurwitz constant for $G$ and it is not isolated in the Markov spectrum $\mathcal{M}_{u}(1,1)$ (see Example 5 in [37]).

Now we shall find $\nu_{\infty}$ in terms of the invariants of $T$. Let $y=\theta+T \theta$. Then by (22) $(x, y)=0$, the points $t, y$, and $x$ lie on the line $(\theta, z)=(T \theta, z)$, and $y \in C_{T}^{\prime}$. We have $y=c(x-(Q(x) /(x, t)) t)$, where $c$ is a nonzero number. Denote $K=Q(x) Q(t) /(x, t)^{2}$. Then

$$
\frac{(y, t)^{2}}{Q(y)}=(K-1) \frac{(x, t)^{2}}{Q(x)}
$$

Let $T \in O(V)$ be a square matrix of order 3 (see Example 5). Assume first that $T$ is not parabolic, i.e. $\lambda \neq 1$. Let $\mu$ and $\mu^{-1}$ be the eigenvalues of $\phi^{-1}(T)$, so that $2 \lambda=\mu+\mu^{-1}$. Let $v, v^{\prime} \in C$ be the eigenvectors of $T$. Then (see (11)) $T v=\mu^{2} v, T v^{\prime}=\mu^{-2} v^{\prime}$. If $(r, t)=0$, then there are numbers $a$ and $b$ such that $r=a v+b v^{\prime}$. We have $(r, r)=2 a b\left(v, v^{\prime}\right), \operatorname{Tr}=a \mu^{2} v+b \mu^{-2} v^{\prime}$ and $(T r, r)=$ $a b\left(\mu^{2}+\mu^{-2}\right)\left(v, v^{\prime}\right)=2 a b\left(2 \lambda^{2}-1\right)\left(v, v^{\prime}\right)$. Hence

$$
(T r+r, r)=2 \lambda^{2}(r, r) .
$$

Now we can find the value of $\nu_{\infty}$ in terms of $\lambda$. Let $x \in C_{T}$. There are numbers $a$ and $b$ such that $x=a t+b r,(t, r)=0$, and $T x=a t+b T r$. We have $(x, t)=$ $a(t, t),(x, x)=a^{2}(t, t)+b^{2}(r, r)$, and $(T x, x)=a^{2}(t, t)+b^{2}(T r, r)$. Thus, by (25), $(T x+x, x)=2 a^{2}(t, t)+2 b^{2} \lambda^{2}(r, r)=0$. Since $a=(x, t) /(t, t), b^{2}(r, r)=$ $(x, x)-(x, t)^{2} /(t, t)$. Hence the equation of $C_{T}$ can be written as follows:

$$
\frac{(x, t)^{2}}{Q(x) Q(t)}=\frac{\lambda^{2}}{\lambda^{2}-1}
$$

Thus $K=1-\lambda^{-2}, \nu_{\infty}=(t, t) / K$, and from (24) we find that

$$
\frac{(y, t)^{2}}{Q(y)}=-\lambda^{-2} \frac{(x, t)^{2}}{Q(x)}
$$

which is true for any $\lambda$. Now from (26) we obtain the equation of $C_{T}^{\prime}$ :

$$
\frac{(y, t)^{2}}{Q(y) Q(t)}=\frac{1}{1-\lambda^{2}} \text {. }
$$

Let $L$ be a line in $D^{2}$ through the point $t$ in $P^{2}$. If $T$ is elliptic and $2 \alpha$ is the angle between $L$ and $T(L)$, then $\lambda=\cos \alpha$ and

$$
\nu_{\infty}=-Q(t) \cot ^{2} \alpha .
$$

If $T$ is hyperbolic and $2 \rho$ is the distance between $L$ and $T(L)$, then $\lambda=\cosh \rho$ and

$$
\nu_{\infty}=Q(t) \operatorname{coth}^{2} \rho \text {. }
$$

Now let $\phi^{-1}(T)=\left(\begin{array}{cc}1 & n \\ 0 & 1\end{array}\right)$. Let $t=(0,0,1)$. Then $T t=t$ and by (8) $\nu_{\infty}=\left|\phi^{-1} T(\eta)-\eta\right|^{2}=n^{2}$ for real $\eta$. 
If $w \neq t$, then

$$
\nu(x)=\nu_{\infty} \frac{(x, w)^{2}}{(x, t)^{2}}
$$

for $x \in C_{T}$. The following statement is clear.

Lemma 20. The largest value of $\nu(x), x \in C_{T}$, is attained at the point of intersection of the line through $w$ and $t$ with $C_{T}$. If this line does not meet the arc of $C_{T}$ with endpoints $u_{1}$ and $u_{2}$, then the maximum and minimum of $\nu(x), x \in \Delta_{F}$, are attained at $u_{1}$ and $u_{2}$.

\section{UNIQUENESS}

Let $G$ be generated by three involutions $S_{1}, S, S_{2}$. Suppose that $\left(S_{1}, S, S_{2}\right)$ is a singular triple - that is, the triangle with vertices $s_{1}, s$, and $s_{2}$ is not obtuse. Let $T=S_{1} S S_{2}$. Let $f_{1}$ and $f_{2}$ be the fixed points of $F_{1}=S S_{2}$ and $F_{2}=S_{1} S$ respectively. Denote by $D_{T}^{\prime}$ the closed region in $A^{2}$ bounded by $C, C_{T}$ and two lines through $w$ and $f_{2}$ and through $w$ and $f_{1}$. Assume that the normalization condition (13) holds. Let

$$
D_{T}=\left\{x \in D_{T}^{\prime}:\left(x, S_{1} w\right) \leq-1 ;\left(x, S_{2} w\right) \leq-1\right\} .
$$

First we shall prove the following.

Theorem 21. Let $f$ be a t-extremal indefinite point in $P^{2}$. If $f \in D_{T}^{\prime}-D_{T}$, then $f=f_{1}$ or $f_{2}$. If $f \in D_{T}$, then $f \in \mathcal{F}(T)$.

For any $w$-extremal $x \in A^{2}$ and any $g \in G$, only one of the following holds:

$$
P(g):(x, g w) \geq 1, \quad \text { or } \quad N(g):(x, g w) \leq-1 .
$$

Let $w=t$. The following statement is analogous to the corollary on p. 32 of [4].

Lemma 22. Let $\left(S_{1}, S, S_{2}\right) \in \Psi(T)$. If $N\left(S_{1}\right), N\left(S_{2}\right), P\left(S^{\prime} T\right)$ and $P\left(S^{\prime \prime} T^{-1}\right)$ hold, then $x=f$.

Proof. We have $S^{\prime} T=F, S^{\prime \prime} T^{-1}=F^{-1}$. Thus, $\left(x, S_{1} w\right) \leq-1,\left(x, S_{2} w\right) \leq$ $-1,\left(x, F^{-1} w\right) \geq 1$ and $(x, F w) \geq 1$, which by Lemma 6] imply $x=f$.

Denote by $R_{T}$ the the interior of $C_{T}$. The following statement is analogous to Lemma 12 on p. 36 of [4].

Lemma 23. Let $\left(S_{1}, S, S_{2}\right) \in \Psi(T)$. If $N\left(S^{\prime}\right)=N\left(S^{\prime} T\right)$ and $N\left(S^{\prime \prime}\right)=N\left(S^{\prime \prime} T^{-1}\right)$ hold, then $x \notin R_{T}$.

Proof. Let $y$ be the point of intersection of $L^{-}\left(S^{\prime}\right)$ and $L^{-}\left(S^{\prime \prime}\right)$. Then $f$ and $y$ lie on the line $L=\left\{x \in A^{2}:\left(x, S^{\prime} t\right)=\left(x, S^{\prime \prime} t\right)\right\}$. It is enough to show that $y \notin R_{T}$. Let $\theta$ be the point of intersection of $M\left(S^{\prime}\right)$ with $C$. Since $s^{\prime \prime}=T^{-1} s^{\prime}$, the point of intersection of $M\left(S^{\prime \prime}\right)$ with $C$ is $T^{-1} \theta$. It is clear that the point $z$ of intersection of the tangent lines to $C$ at $\theta$ and $T^{-1} \theta$ lies on $L$. On the other hand, by definition $z \in C_{T}$. The lemma follows from the fact that $z$ lies on $L$ between $f$ and $y$.

Let $\left(S_{1}, S, S_{2}\right)$ be one of the singular triples of involutions. Let $S^{\prime}=S_{1} S S_{1}$ and $S^{\prime \prime}=S_{2} S S_{2}$. It was shown in Sec. 4 that the fixed points $s^{\prime}, s_{1}$, $s_{2}$, of these involutions belong to the boundary of $D^{*}(t)$. By Lemma $8 f_{2}, f, f_{1}$, the fixed points of $F_{2}, F, F_{1}$, as well as $f_{1}^{\prime}$ and $f_{2}^{\prime}$, the fixed points of $F_{1}^{\prime}=S^{\prime} S_{2}$ and $F_{2}^{\prime}=S_{1} S^{\prime \prime}$, are $t$-extremal. The triple $\left(S^{\prime}, S_{1}, S_{2}\right)$ is a nonsingular neighbor of $\left(S_{1}, S, S_{2}\right)$ (see Fig. 5). 
Denote by $R^{\prime \prime}=R\left(f_{2}, f\right)$ the region in $D_{T}^{\prime}-D^{*}(t)$ bounded by two lines through $f$ and $s$ and through $f_{2}$ and $s_{2}^{\prime}$, the fixed point of $S_{2}^{\prime}=S_{1} S_{2} S_{1}$. By Lemma 12] $u$ and $u^{\prime}=S_{1} u$ are vertices of $R^{\prime \prime}$; hence $\left[u, u^{\prime}\right] \subset L^{+}\left(S_{1}\right)$ is a common side of $R^{\prime \prime}$ and $D^{*}(t)$. Thus, any indefinite $t$-extremal $x \in R^{\prime \prime}$ satisfies $N\left(S_{1}\right)$. Similarly, let $R^{\prime}=R\left(f, f_{1}\right)$ be the region in $D_{T}^{\prime}-D^{*}(t)$ bounded by two lines through $f_{1}$ and $s_{1}^{\prime \prime}$, the fixed point of $S_{2} S_{1} S_{2}$, and through $f$ and $s$. Then any indefinite $t$ extremal $x \in R^{\prime}$ satisfies $N\left(S_{2}\right)$. Let $R=R^{\prime} \cup R^{\prime \prime}$. Thus, if $x \in R$ satisfies $P\left(S_{2}\right)=P\left(S_{2} T^{-1}\right)=P\left(F_{2}^{-1}\right)$ and $N\left(S_{1}\right)$, then $x=f_{2}$. If $x \in R$ satisfies $P\left(S_{1}\right)=$ $P\left(S_{1} T\right)=P\left(F_{1}\right)$ and $N\left(S_{2}\right)$, then $x=f_{1}$. For all other $x \in R$,

$$
N\left(S_{2}\right) \text { and } N\left(S_{1}\right)
$$

hold simultaneously. Hence $x \in D_{T}$.

Now Theorem 21 follows from Lemma 24 below, which is analogous to Lemma 13 on p. 36 of 4 .

Lemma 24. Assume that $x \in D_{T}$. If $x$ is t-extremal, then $x \in \mathcal{F}(T)$.

Proof. The triples $\left(S^{\prime}, S_{1}, S_{2}\right)$ and $\left(S_{1}, S_{2}, S^{\prime \prime}\right)$, where $S^{\prime}=S_{1} S S_{1}$ and $S^{\prime \prime}=S_{2} S S_{2}$, are the nonsingular neighbors of $\left(S_{1}, S, S_{2}\right)$ (see Fig. 5). If

$$
P(F)=P\left(S^{\prime} T\right) \quad \text { and } \quad P\left(F^{-1}\right)=P\left(S^{\prime \prime} T^{-1}\right)
$$

hold simultaneously, then $x=f$ by Lemma 22 Otherwise, either

$$
N\left(S^{\prime}\right) \text { and } N\left(S_{2}\right)
$$

hold simultaneously or

$$
N\left(S^{\prime \prime}\right) \text { and } N\left(S_{1}\right)
$$

hold simultaneously, which are (27) for the triples $\left(S^{\prime}, S_{1}, S_{2}\right)$ and $\left(S_{1}, S_{2}, S^{\prime \prime}\right)$ respectively. It is clear now that the proof can be completed by induction.

Example 25. Let $d$ be a square-free integer with $d \equiv 1(\bmod 4)$. Let $\omega=$ $(1+\sqrt{-d}) / 2$. Let $\mathcal{O}_{d}$ be the ring of integers in the imaginary quadratic field $\mathbf{Q}(\sqrt{-d})$. It is shown in [38] that when $d=3,7$ or 11 the stabilizer of the line $\omega \mathbf{R} \subset \mathbf{C}$ in $G L_{2}\left(\mathcal{O}_{d}\right)$ is isometric to the Hecke group $G_{q}, q=3,4$ or 6 , respectively. When $d=19$, the stabilizer of $\omega \mathbf{R}$ is isometric to the group $G$ generated by reflections

$$
\sigma=\left(\begin{array}{cc}
-1 & 0 \\
0 & 1
\end{array}\right), \sigma_{0}=\left(\begin{array}{ll}
0 & 1 \\
1 & 0
\end{array}\right), \sigma_{1}=\left(\begin{array}{cc}
-1 & \sqrt{5} \\
0 & 1
\end{array}\right), \sigma_{2}=\left(\begin{array}{cc}
\sqrt{5} & -2 \\
2 & -\sqrt{5}
\end{array}\right)
$$

in the sides of the quadrangle with vertices at $\infty$ and at the centers of involutions $S_{1}=\sigma \sigma_{0}, S=\sigma_{0} \sigma_{2}$, and $S_{2}=\sigma_{2} \sigma_{1}$. Thus,

$$
S_{1}=\left(\begin{array}{cc}
0 & -1 \\
1 & 0
\end{array}\right), \quad S=\left(\begin{array}{cc}
2 & -\sqrt{5} \\
\sqrt{5} & -2
\end{array}\right), \quad S_{2}=\sigma_{2} \sigma_{1}=\left(\begin{array}{cc}
-\sqrt{5} & 3 \\
-2 & \sqrt{5}
\end{array}\right) .
$$

By Lemma $8 f_{2}$ and $f_{1}$, the fixed points of $F_{2}=S_{1} S$ and $F_{1}=S S_{2}$, are $w$ extremal. Let $\Psi(T), T=S_{1} S S_{2}=\sigma \sigma_{1}=\left(\begin{array}{cc}1 & -\sqrt{5} \\ 0 & 1\end{array}\right)$, be the tree of triples of involutions generated by the triple $\left(S_{1}, S, S_{2}\right)$. Thus $w=t$ and $\lambda=1$. The tree of indefinite points $\mathcal{F}(T)$ associated with $\Psi(T)$ is extremal. By Theorem 21] if $\nu(f)<5$ for an indefinite $f \in P^{2}$, then $g(f) \in \mathcal{F}(T)$ for some $g \in G$. If we put $m_{2}=\sqrt{5} x_{2}, m_{1}=\sqrt{5} x_{1}, m=5 x$ in (17), then it is reduced to the equation $x_{2}^{2}+x_{1}^{2}+5 x^{2}=5 x_{2} x_{1} x, x_{2}, x_{1}, x \in \mathbf{Z}$. This equation first appeared in [29], where it 
was used to describe the discrete part of the Markov spectrum on the sublattice of index 5 (see also [19]). Moreover the Markov spectrum for $G$ above $5^{-1 / 2}$, the first accumulation point of the spectrum, can be also described in terms of the solutions of this equation. In particular the first two minima are 1 and $1 / 2$. It follows from results obtained in [28, that the Markov spectrum for $G$ is a subset of the Markov spectrum for $G L_{2}\left(\mathcal{O}_{19}\right)$. Thus $5^{-1 / 2}$ is an accumulation point in the spectrum. Note that 1 is the approximation constant for the imaginary quadratic field with discriminant -19 (see e.g. [35]).

Similarly, it can be shown that the Markov spectrum of the Hecke group $G_{4}$ is a subset of the Markov spectrum for $G L_{2}\left(\mathcal{O}_{7}\right)$. In that case the equation $x_{2}^{2}+$ $x_{1}^{2}+2 x^{2}=4 x_{2} x_{1} x$ can be used to show that $8^{-1 / 2}$ is an accumulation point in the spectrum. This equation first appeared in 27. It can be used to obtain the complete description of the discrete part of the Markov spectrum on the sublattice of index 2 (see [19]).

Example 26. (see [39]). Suppose that $G$ is generated by the reflections $\sigma_{0}, \sigma_{1}$ and $\sigma$ across the sides $s v, v w$ and $s w$ of the triangle with vertices $s, v$ and $w$. Let

$$
S=\sigma \sigma_{0}=\sigma_{0} \sigma, \quad A=\sigma_{1} \sigma_{0}, \quad B=A S=\sigma_{1} \sigma,
$$

and $S^{2}=A^{q}=B^{m}=\mathrm{id}$, where $q=3$ or 4 . Thus, $w=B w$ is an elliptic fixed point of the subgroup $G^{\prime \prime}$ of $G$ generated by $S$ and $A$. $G^{\prime \prime}$ is called the triangle group $(2, q, m)$ (see e.g. [2]).

Let $S_{k}=A^{k} S A^{-k}$ and $S_{k}^{\prime}=S S_{k} S, k=1,2$. When $q=4, U=A^{2}$ is also an involution. Let $U^{\prime}=S U S$. If $q=4$, let $T=S_{1} S U^{\prime}$ and let $\Psi(T)$ be the tree of involutions generated by the triple $\left(S_{1}, S, U^{\prime}\right)$. For $q=3, T=S_{1} S S_{2}^{\prime}$ and $\Psi(T)$ is the tree generated by the triple $\left(S_{1}, S, S_{2}^{\prime}\right)$. Then $T=B^{q-6},(q=3,4), w$ is the fixed point of $T$ and, therefore, the equation of $C_{T}$ in $A^{2}$ is $Q(x)=$ const. It is shown in [39] that the corresponding tree $\mathcal{F}(T)$ of indefinite points in $P^{2}$ is unique in $D_{T}$.

Let $H$ be a Fuchsian group and let $G$ be its subgroup generated by three involutions $S_{1}, S$ and $S_{2}$. Let $\left(S_{1}, S, S_{2}\right)$ be a nonsingular triple. Let $T=S_{1} S S_{2}$. If $w \neq t$ then, in general, the $g$-strip and $g T^{n}$-strip do not coincide. Arguing as in the proof of Theorem 21, we obtain the following.

Lemma 27. Assume that for any triple $\left(S_{1}, S, S_{2}\right) \in \Psi(T)$ there are compound $\left(S_{2}, S_{2} T^{-1}\right)$-, $\left(S_{1}, S_{1} T\right)-,\left(S^{\prime}, S^{\prime} T\right)$ - and $\left(S^{\prime \prime}, S^{\prime \prime} T^{-1}\right)$-strips in $D_{T}^{\prime}$. If $f \in D_{T}^{\prime}$ is a w-extremal indefinite point, then $f=f_{1}$, or $f=f_{2}$, or $f \in \mathcal{F}(T)$.

The rest of this section is devoted to the case when $T$ is hyperbolic and $w$ is isotropic. The results obtained are applicable to zonal Fuchsian groups. In particular, in the next section we shall discuss their application to the maximal Fuchsian groups commensurable with the modular group. As in Example 5 we shall identify the points $f=(\alpha, \beta, \gamma) \in P^{2}$ with the quadratic forms $f(x, y)=$

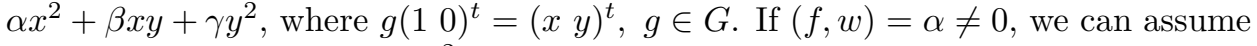
that $\alpha=1$. Then $Q(f)=\beta^{2}-4 \gamma$. A form $f$ is extremal iff $|f(x, y)| \geq 1$ for all $g \in G$. The $g$-strip is the strip $\left|x^{2}+\beta x y+\gamma y^{2}\right|<1$ in the $(\beta, \gamma)$-plane.

Let $g, g^{\prime} \in G, g\left(\begin{array}{ll}1 & 0\end{array}\right)^{t}=\left(\begin{array}{ll}x & y\end{array}\right)^{t}, g^{\prime}\left(\begin{array}{ll}1 & 0\end{array}\right)^{t}=\left(\begin{array}{ll}x^{\prime} & y^{\prime}\end{array}\right)^{t}$, and let $z=x / y, z^{\prime}=x^{\prime} / y^{\prime}$. Let $f$ be the fixed point of a hyperbolic element $F \in G$. Assume that

$$
\theta<z<z^{\prime}<\theta^{\prime}
$$


where $f(\theta, 1)=f\left(\theta^{\prime}, 1\right)=0$ and $F^{i}(x) \rightarrow \theta^{\prime}$ as $i \rightarrow \infty$ for any $x \in C, x \neq \theta$. Let $\Phi=\left(\begin{array}{ll}a & b \\ c & d\end{array}\right) \in \operatorname{Stab}(f, O(V))$, so that $z^{\prime}=\Phi(z)=(a z+b) /(c z+d)$.

Let $F^{i} g\left(\begin{array}{ll}1 & 0\end{array}\right)^{t}=\left(\begin{array}{ll}x_{i} & y_{i}\end{array}\right)^{t}, F^{i} g^{\prime}\left(\begin{array}{ll}1 & 0\end{array}\right)^{t}=\left(x_{i}^{\prime}, y_{i}^{\prime}\right)^{t}, z_{i}=x_{i} / y_{i}, z_{i}^{\prime}=x_{i}^{\prime} / y_{i}^{\prime}, \quad i=$ $1,2, \ldots$ Denote by $h_{i}$ the point of intersection of $L^{-}\left(F^{i} g\right)$ and $L^{+}\left(F^{i} g^{\prime}\right), i=$ $1,2, \ldots$ In particular, $h=h_{0}=(1, \beta, \gamma)$ is the point of intersection of $L^{-}(g)$ with $L^{+}\left(g^{\prime}\right)$. For indefinite $f \in P^{2}$ and a real number $z$, we temporarily abbreviate $f(z)=f(z, 1)$. Let

$$
k=h(z) / f(z), \quad k^{\prime}=h\left(z^{\prime}\right) / f\left(z^{\prime}\right) .
$$

Then $z_{i}^{\prime}=\Phi\left(z_{i}\right), k=h_{i}\left(z_{i}\right) / f\left(z_{i}\right), k^{\prime}=h_{i}\left(z_{i}^{\prime}\right) / f\left(z_{i}^{\prime}\right), i=1,2, \ldots$

Lemma 28. The indefinite points $h_{i}, i=1,2, \ldots$, lie on the hyperbola

$$
\begin{aligned}
& {\left[h(-d, c)-\left(k+k^{\prime}-1\right)|\Phi|\right]\left[h(a, c)-\left(k+k^{\prime}-1\right)|\Phi|\right]} \\
& \quad=(k-1)\left(k^{\prime}-1\right)(a+d)^{2}|\Phi|
\end{aligned}
$$

where $|\Phi|=\operatorname{det} \Phi$.

The sequence $Q\left(h_{i}\right), i=1,2, \ldots$, is increasing.

Proof. Denote $r=c z+d$. We have $f\left(z^{\prime}\right)=|\Phi| r^{-2} f(z), c^{2} f(z)=r^{2}-(a+d) r+|\Phi|$, and $z-z^{\prime}=c f(z) / r$. Here $c f=(c, d-a,-b)$. Hence $h(z)-h\left(z^{\prime}\right)=\left(z-z^{\prime}\right)\left(\beta+z+z^{\prime}\right)$ and $k f(z)-k^{\prime} f\left(z^{\prime}\right)=c f(z)\left(\beta+z+z^{\prime}\right) / r$. Thus,

$$
\beta(h)=-\left(z+z^{\prime}\right)+\left(k r-k^{\prime} r^{-1}|\Phi|\right) / c, \quad \gamma(h)=z z^{\prime}-\left(k r z^{\prime}-k^{\prime} r^{-1} z|\Phi|\right) / c .
$$

For $\Phi, k$ and $k^{\prime}$ fixed, the equations obtained are the parametric equations in $z$ of a hyperbola. Indeed, eliminating $z$, we obtain (28), which is an equation of a hyperbola with asymptotes $h(-d, c)=\left(k+k^{\prime}-1\right)|\Phi|, h(a, c)=\left(k+k^{\prime}-1\right)|\Phi|$. One can easily verify that $Q(h)=\beta^{2}-4 \gamma$ attains maximum at $\beta=(d-a) / c$. Evidently the points $h_{i}$ lie on this curve, and the sequence $\left|\beta\left(h_{i}\right)-(d-a) / c\right|$ is decreasing.

Remark. It is clear that Lemma 28 holds in the case when $F^{i}(x) \rightarrow \theta$ as $i \rightarrow \infty$ for any $x \in C, x \neq \theta^{\prime}$.

Let $m_{T}=\sup Q(f)$, the supremum being taken over all $f \in D_{T}$. Denote

$$
D_{T}^{\prime \prime}=\left\{x \in D_{T}^{\prime}: Q(x)<m_{T}\right\} .
$$

The following theorem is very important for applications, because it reduces the problem of uniqueness of the tree $\mathcal{F}(T)$ in $D_{T}$ to the verification of existence of only two compound strips in $D_{T}^{\prime \prime}$.

Theorem 29. Let $w \in C$. Assume that there exist compound $\left(S_{2}, S_{2} T^{-1}\right)$ - and $\left(S_{1}, S_{1} T\right)$-strips in $D_{T}^{\prime \prime}$. Let $f \in D_{T}^{\prime}$ be a w-extremal indefinite point. If $f \in$ $D_{T}^{\prime}-D_{T}$, then $f=f_{1}$ or $f_{2}$. If $f \in D_{T}$, then $f \in \mathcal{F}(T)$.

Proof. By Lemma 27 it is enough to show that for any triple of involutions $\left(S_{1}, S, S_{2}\right) \in \Psi(T)$ the compound $\left(S^{\prime}, S^{\prime} T\right)$ - and $\left(S^{\prime \prime}, S^{\prime \prime} T^{-1}\right)$-strips in $D_{T}^{\prime \prime}$ exist provided that the compound $\left(S_{2}, S_{2} T^{-1}\right)$ - and $\left(S_{1}, S_{1} T\right)$-strips in $D_{T}^{\prime \prime}$ exist.

The triples $\left(S^{\prime}, S_{1}, S_{2}\right)$ and $\left(S_{1}, S_{2}, S^{\prime \prime}\right)$ are the neighbors of $\left(S_{1}, S, S_{2}\right)$ in $\Psi(T)$ with larger height. Here $S^{\prime}=F_{2} S_{1}=S_{1} S S_{1}$ and $S^{\prime \prime}=F_{1}^{-1} S_{2}=S_{2} S S_{2}$. Assume that the compound $\left(S_{2}, S_{2} T^{-1}\right)$-strip in $D_{T}^{\prime \prime}$ consists of the $g_{i}$-strips, $0 \leq i \leq$ $k, g_{0}=S_{2}, g_{k}=S_{2} T^{-1}$. Then, by Lemma [28] the $F_{1}^{-1} g_{i}$-strips, $0 \leq i \leq k$, form a compound $\left(S^{\prime \prime}, S^{\prime \prime} T^{-1}\right)$-strip in $D_{T}^{\prime \prime}$. Similarly, if the compound $\left(S_{1}, S_{1} T\right)$-strip 
in $D_{T}^{\prime \prime}$ consists of the $g_{i}$-strips, $0 \leq i \leq k, g_{0}=S_{1}, g_{k}=S_{1} T$, then the $F_{2} g_{i^{-}}$ strips, $0 \leq i \leq k$, form a compound $\left(S^{\prime}, S^{\prime} T\right)$ - strip in $D_{T}^{\prime \prime}$. Now the proof can be completed by induction.

\section{Markov SPECTRUM on SUblattices}

Let $m$ be a positive integer. Denote by $\mathcal{F}_{m}$ the set of indefinite binary quadratic forms $f$ satisfying the following condition:

$$
|f(x, y)| \geq \operatorname{gcd}(x, m) \mu(f),
$$

where $\mu(f)=\inf |f(x, y)|$, the infimum being taken over all $(x, y) \in \mathbf{Z}^{2} /(0,0)$. The subset

$$
\mathcal{M}_{m}=\left\{\nu_{w}(f): f \in \mathcal{F}_{m}\right\}
$$

of the classical Markov spectrum $\mathcal{M}$ (see Example 5) is called the Markov spectrum on the sublattice of index $m$ (see [19]). For $m=2,5,6$, the discrete part of that spectrum was found in [27], 29], 30] respectively, and for $m=3$, in 24] (see also Example 30 below).

Let $m$ be square-free and

$G^{\prime}(m)=\left\{g=\left(\begin{array}{ll}a & b \\ c & d\end{array}\right) \in M_{2}(\mathbf{Z}) \mid b \equiv 0(\bmod m), a \equiv d \equiv m \equiv 0 \quad(\bmod \operatorname{det} g)\right\}$.

Let $G=P G^{\prime}(m), G_{o}=G \cap P G L_{2}(\mathbf{Z})$. Then $G_{o}$ is normal in $G$ and $\left[G: G_{o}\right]=2^{t}$, where $t$ is the number of prime divisors of $m$ (see [16]). It follows from the definition of $G^{\prime}(m)$ that

$$
\operatorname{gcd}(a, m)=\operatorname{det} g .
$$

In what follows, we employ the usual abuse of language and refer to the elements of $G$ as matrices by setting

$$
G(m)=\left\{|\operatorname{det} g|^{-1 / 2} g: g \in G^{\prime}(m)\right\} .
$$

Remark. Let $G_{q}$ be the Hecke group. Let $\tau=\left(\begin{array}{cc}\sqrt{m} & 0 \\ 0 & 1\end{array}\right)$. It can be easily verified that, for $m=2$ and $3, G(m)=\tau G_{2 m} \tau^{-1}$. In [39], the discrete parts of the Markov spectra for the Hecke groups $G_{q}, q \geq 5$, are found. These results can be used to describe the spectrum on the sublattice of index $m=3$ (see Example 30).

Let $w=(1,0,0)$. Assume that an indefinite point $f \in A^{2}$ is $w$-extremal; that is, $|f(x, y)| \geq 1$ for any $g \in G(m),(x y)^{t}=g(10)^{t}$. By (29) and (30), these conditions are equivalent to following: for any integers $x$ and $y$,

$$
|f(x, y)| \geq \operatorname{gcd}(x, m) \text {. }
$$

Thus the Markov spectrum of $G(m)$ coincides with the Markov spectrum on the sublattice of index $m$ for the forms $f$ with attainable minimum (see [19]).

Let $S_{1}, S, S_{2} \in G^{\prime}(m)$ be involutions. Let $k_{1}=\operatorname{det} S_{1}, k=\operatorname{det} S$. It can easily be shown that

$$
c^{2}\left(S_{1} S\right)+k_{1} c^{2}(S)+k c^{2}\left(S_{1}\right)=-\operatorname{tr}\left(S_{1} S\right) c(S) c\left(S_{1}\right),
$$

where $\operatorname{tr}\left(S_{1} S\right) \equiv 0\left(\bmod k k_{1}\right)$. Let $F_{2}=S_{1} S=T S_{2}$. Assume that $T$ is parabolic. Then $\operatorname{tr}\left(S_{1} S\right) \equiv 0\left(\bmod k k_{1} c\left(S_{2}\right)\right)$. Hence

$$
c^{2}\left(S_{2}\right)+k_{1} c^{2}(S)+k c^{2}\left(S_{1}\right)=n k k_{1} c\left(S_{2}\right) c(S) c\left(S_{1}\right)
$$


for some integer $n$. It is proved in [31] (see also [22]) that, up to permutation of $k$ and $k_{1}$, this Diophantine equation has a solution in integers $\left(c\left(S_{2}\right), c(S), c\left(S_{1}\right)\right) \neq$ $(0,0,0)$ only for $\left(n, k, k_{1}\right)=(3,1,1),(2,2,1),(1,2,2),(1,2,3),(1,1,5),(1,1,1)$, $(1,2,1)$. It follows that there is a tree of involutions $\Psi(T)$ with parabolic $T$ only for $m=1,2,5$ and 6 . As was mentioned above, for $m=2,5$ and 6 , the discrete part of the Markov spectrum $\mathcal{M}_{m}$ was found in [27, [29, [30]. For any other value of $m, T$ can only be hyperbolic. The results obtained in the preceding sections can be used to find the discrete part of $\mathcal{M}_{m}$ in those cases. In the examples below, we shall identify the elements of $G=P G^{\prime}(m)$ with the corresponding elements in $G^{\prime}(m)$. Let

$$
\sigma=\left(\begin{array}{cc}
-1 & 0 \\
0 & 1
\end{array}\right), \quad \sigma_{0}(m)=\left(\begin{array}{cc}
0 & m \\
1 & 0
\end{array}\right), \quad \sigma_{1}(m)=\left(\begin{array}{cc}
-1 & m \\
0 & 1
\end{array}\right) .
$$

Example 30. Let $m=3$. As was mentioned above, the group $G$ is conjugate in $G L_{2}(\mathbf{R})$ to the Hecke group $G_{6}$. It is generated by reflections $\sigma, \sigma_{0}=\sigma_{0}(3), \sigma_{1}=$ $\sigma_{1}(3)$. Let $S=\sigma_{0} \sigma, A=\sigma_{1} \sigma_{0}, U=A^{3}, U^{\prime}=S U S, S_{k}=A^{k} S A^{-k}, S_{k}^{\prime}=$ $S S_{k} S, k=0,1, \ldots, 5$. Then $S^{2}=S_{k}^{2}=A^{6}=$ id. Let $f_{k}$ be the fixed point of $F_{k}=S_{k} S, k=1,2,3$, and let $K_{3}=S U^{\prime}$. Then $F_{3}=K_{3}^{2}$. The points $f_{k}$ are extremal (see [39]). Let $\Psi\left(T_{2}\right)$ and $\Psi\left(T_{3}\right)$ be the trees of triples of involutions generated by the triples $\left(S_{1}, S, S_{2}^{\prime}\right)$ and $\left(S_{2}, S, U^{\prime}\right), T_{2}=S_{1} S S_{2}, T_{3}=S_{2} S U^{\prime}$, and let $\mathcal{F}\left(T_{2}\right)$ and $\mathcal{F}\left(T_{3}\right)$ be the corresponding trees of extremal points. It is shown in [39] that:

1) The tree $\mathcal{F}\left(T_{3}\right)$ is unique in the region $D_{3}$ bounded by the axes of involutions $U^{\prime}$ and $S_{2}$ and the hyperbola $C_{T}$ with equation $\left(T_{3} x+x, x\right)=0$ in $P^{2}$; that is, if an extremal $f \in D_{3}$, then $f \in \mathcal{F}\left(T_{3}\right)$.

2) Let $G_{S}$ be the subgroup of $G$ generated by $S_{k}, k=0,1,2$, and $U$. Then the trees $\mathcal{F}\left(T_{2}\right)$ and $\mathcal{F}\left(T_{3}\right)$ both are simple on $M=D^{2} / G_{S}$; that is, the projections of the polars of points in these trees into $M$ are simple closed geodesics on $M$.

3) The first limit point in the Markov spectrum of $G$ is $1 / 4$ and the discrete part of the spectrum if found (cf. [24]). The first limit point of $\mathcal{F}\left(T_{2}\right)$ in the spectrum is $1 / 5$.

Example 31. Let $m=13$. The group $G$ is generated by reflections $\sigma, \sigma_{0}(13)$, $\sigma_{1}(13)$, and

$$
\sigma_{2}=\left(\begin{array}{cc}
13 & -78 \\
2 & -13
\end{array}\right), \quad \sigma_{3}=\left(\begin{array}{cc}
13 & -52 \\
3 & -13
\end{array}\right)
$$

Suppose, as we can, that $f=(1, \beta, \gamma) \in A^{2}$ satisfies the condition $-13 \leq \beta \leq 0$.

If $N\left(\sigma_{0}\right)$ holds, then $Q(f) \geq 52$. If $N\left(\sigma_{2}\right)$ holds, then $Q(f) \geq 13$. Denote by $R$ the set of $f \in A^{2}$ for which $N\left(\sigma_{3}\right), P\left(\sigma_{0}\right)$ and $P\left(\sigma_{2}\right)$ hold. Then

$$
Q(f) \leq 12
$$

for any $f \in R$, and 12 is attained at $t_{0}=(1,-8,13)$, the fixed point of $T_{o}=\sigma_{2} \sigma_{0}=$

$$
\begin{aligned}
& \left(\begin{array}{cc}
6 & -13 \\
1 & -2
\end{array}\right) \text {. Let } \\
& \quad S_{1}=\left(\begin{array}{cc}
70 & -169 \\
29 & -70
\end{array}\right), \quad S=\left(\begin{array}{cc}
5 & -13 \\
2 & -5
\end{array}\right), \quad S_{2}=\left(\begin{array}{cc}
57 & -325 \\
10 & -57
\end{array}\right) .
\end{aligned}
$$

Direct verification shows that if $f(5,2) \geq 1$, then $|f(3,1)|<1$ or $|f(13,5)|<13$. Thus, for any extremal $f \in R, N(S)$ holds. Let $\Psi(T)$ be the tree of triples of 
involutions generated by $\left(S_{1}, S, S_{2}\right)$, where $T=S_{1} S S_{2}=T_{o}^{3}=\left(\sigma_{2} \sigma_{0}\right)^{3}$. The fixed points of

$$
F_{2}=S_{1} S=\left(\begin{array}{cc}
12 & -65 \\
5 & -27
\end{array}\right), \quad F_{1}=S S_{2}=\left(\begin{array}{cc}
155 & -884 \\
64 & -365
\end{array}\right)
$$

are $f_{2}=(5,-39,-65)$, the third Markov form (see 4], Chapter II), and $f_{1}=$ $(16,-130,221)$. They are extremal, (the period of the continued fraction expansion of a root of $f_{1}$ is $(\overline{2,1,2,2,1,2,1,1})$ ). Note that $\sigma_{0} f_{2}=f_{2}$ and $\sigma_{2} f_{1}=f_{1}$. By Theorem 18, all the indefinite points in the tree $\mathcal{F}(T)$ are extremal and all its limit points lie on the hyperbola $C_{T}$ with equation (23).

Now we shall prove that, inside $C_{T}$, the tree obtained is unique. Let $g_{1}, g_{2} \in$ $G, g_{i}(1,0)^{t}=\left(a_{i}, c_{i}\right)^{t}$. The point of intersection of $L^{+}\left(g_{1}\right)$ and $L^{-}\left(g_{2}\right)$ is the fixed point $h$ of $S \sigma$, where

$$
\begin{array}{cc}
S=\left(\begin{array}{cc}
a_{2} & -b_{2} \\
c_{2} & -a_{2}
\end{array}\right), & \sigma=\left(\begin{array}{cc}
a_{1} & -b_{1} \\
c_{1} & -a_{1}
\end{array}\right), \\
b_{1}=\left(a_{1}^{2}+1\right) / c_{1}, & b_{2}=\left(a_{2}^{2}-1\right) / c_{2} .
\end{array}
$$

Thus,

$$
Q(h)=c^{-2}\left(r^{2}(h)+4\right)
$$

where $c=c(h)=a_{1} c_{2}-a_{2} c_{1}$ and, by (31), tr $h=\left(c_{1} c_{2}\right)^{-1}\left(c_{2}^{2}-c_{1}^{2}-c^{2}\right)$. In particular,

$$
Q(h)>4 c^{-2} .
$$

There are compound $\left(S_{2}, S_{2} T^{-1}\right)$ - and $\left(S_{1}, S_{1} T\right)$-strips in $R$ which consist of $S_{2}$, $S_{2} \sigma_{0}, S_{2} T_{o}^{-1}, S_{2} T_{o}^{-1} \sigma_{0}, S_{2} T_{o}^{-2}, S_{2} T_{o}^{-2} \sigma_{0}, S_{2} T^{-1}$-strips and of $S_{1}, S_{1} \sigma_{2}, S_{1} T_{o}$, $S_{1} T_{o} \sigma_{2}, S_{1} T_{o}^{2}, S_{1} T_{o}^{2} \sigma_{2}, S_{2} T$-strips respectively. This follows from (32) and (33), since the sequence of values of $c^{-2}(h)$ for the pairs of adjacent strips is $13 / 4,13$, $13 / 4,13,13 / 4,13$ for the compound $\left(S_{2}, S_{2} T^{-1}\right)$-strip and 13,13/4,13,13/4, 13, $13 / 4$ for the $\left(S_{1}, S_{1} T\right)$-strip. Thus the uniqueness of the tree $\mathcal{F}(T)$ of extremal indefinite points in $R$ follows from Theorem 29. Let $u_{i}$ be the point of intersection of the tangent line to the absolute $C$ through $f_{i}, i=1,2$, with $C_{T}$. The limit set of $\mathcal{F}(T)$ is located on the hyperbola $C_{T}$ between the points $u_{1}$ and $u_{2}$. The roots of $u_{i}(x, 1)=0$ are $\theta_{i}$ and $S_{i}\left(\theta_{i}\right)$, where $f_{i}\left(\theta_{i}, 1\right)=0, i=1,2$, and $\theta_{1}=(65+$ $\sqrt{689}) / 16=5.703050594, \theta_{2}=(39-\sqrt{221}) / 10=2.413393125$. Thus, the values of $\nu_{w}(x)$, where $x$ runs through the limit set of $C_{T}$, form a Cantor set $U$ in the classical Markov spectrum $\mathcal{M}$, and $U \subset\left[\nu_{w}\left(u_{2}\right), \nu_{w}\left(u_{1}\right)\right]=[0.303983697,0.303986571]$. Note that the vertical line through $t_{o}$ does not intersect the interval $\left[u_{2}, u_{1}\right]$, and $\nu_{w}(x)$ is an increasing function on this interval.

Let $f^{(k)}$ be the indefinite fixed point of $F_{2}^{k} F_{1}, k=0,1, \ldots$, so that $f^{(0)}=f_{1}$ and $S_{2} f^{(k)}=-f^{(k)}$. Applying the approach developed in Example 7 of [39], we obtain the following.

Theorem 32. Let $G=G(13)$. Let $f$ be an indefinite point such that $\nu(f)>\nu\left(u_{2}\right)$. Then $f$ is $G$-equivalent to $f_{2}$ or one of the points $f^{(k)}, k=0,1, \ldots$

Remark. By the isolation theorem (see [4], p. 25), which holds for any zonal Fuchsian group, all the $f \in \mathcal{F}(T)$ are locally isolated; that is, there is a constant $\epsilon>0$ such that $\nu_{w}(f)-\epsilon>\nu_{w}\left(f^{\prime}\right)$ for any $f^{\prime} \in A^{2}$ which is sufficiently close to $f$. This theorem can be also applied to show that the results obtained hold for the indefinite forms with unattainable minimum as well (see 4], p. 40). 


\section{Simple Closed GeOdesics}

Let $G$ be a discrete group. The quotient $M=D^{2} / G$ is a Riemann surface. Let $\pi: D^{2} \rightarrow D^{2} / G$ be the projection map. If $L$ is the axis of a hyperbolic element $F \in G$, then $\pi(L)$ is a closed geodesic in $M$, and if $\gamma$ is a closed geodesic in $M$, then $\pi^{-1}(\gamma)$ consists of the axes of the conjugacy class of some hyperbolic $F \in G$. A geodesic $\gamma$ is said to be simple if it does not intersect itself. Let $f \in P^{2}$ be the pole of $L$. It is easily seen that

$$
\pi(L) \text { is simple iff } L \cap g L=\emptyset \text { for all } g \in G / G_{f} .
$$

(or iff the line through $f$ and $g(f)$ in $P^{2}$ cuts $C$ for all $g \in G / G_{f}$ ). Roughly speaking, this means that the $G$-orbit of the indefinite point $f$ in $P^{2}$ must be "close" to $C$ if $\pi(L)$ is a simple geodesic in $M$.

Let $G$ be generated by three involutions $S_{1}, S, S_{2}$. Suppose that the triangle with vertices $s_{1}, s$, and $s_{2}$ is not obtuse. Let $T=S_{1} S S_{2}$. Applying Theorem 21 we shall prove the following.

Theorem 33. Let $G$ be generated by three involutions $S_{1}, S, S_{2}$. Assume that

$$
(T f-f, f)<0 \text {. }
$$

A geodesic $\gamma=\pi(L)$ in $M=D^{2} / G$ is simple if and only if the pole $f$ of $L$ is $G$-equivalent to $f_{1}, f_{2}$, or a point in the tree $\mathcal{F}(T)$ or its closure.

If $T$ is hyperbolic and $L_{T}$ is the axis of $T$, then $\pi\left(L_{T}\right)$ is also simple in $M$.

Proof. First assume that

$$
\delta(f)=(T f, f)^{2}-(f, f)^{2}<0,
$$

i.e. $f \notin \bar{R}_{T}$, where $R_{T}$ is the interior of $C_{T}$. The line $x=a f+b T f$ cuts $C$ if and only if the discriminant $\delta(f)$ of the quadratic form $(x, x)$ is positive. Thus, if $f \notin \bar{R}_{T}$ then the line through $f$ and $T f$ does not cut $C$; hence the lines $L$ and $T(L)$ meet in $D^{2}$ and the geodesic $\gamma$ in $M$ is not simple.

Now let $G f \in R_{T}$. By Theorem 21 we can assume that $f \in \mathcal{F}(T)$, since the projections of the polars of $f_{1}$ and $f_{2}$ into $M$ are simple (see Fig. 4). Let $\left(S_{1}, S, S_{2}\right), T=S_{1} S S_{2}$, be the associated triple of involutions, so that $G_{f}=$ $\left\langle S_{1}, S_{2}\right\rangle$. Then the interval $\left[s_{1}, s_{2}\right)$ is a fundamental domain of $G_{f}$ on $L$, the polar of $f$. Let $T t=t, t^{\prime}=S_{1} t$ and $t^{\prime \prime}=S_{2} t=S t^{\prime}$. Then the triangle $\Delta$ with vertices at $t, t^{\prime}$ and $t^{\prime \prime}$ is a fundamental domain of $G$. Since $\left[s_{1}, s_{2}\right) \subset \Delta$, the geodesic $\gamma=\pi(L)$ is simple in $M$, as required.

Let $L$ be the line through the points $t$ and $s^{\prime \prime}$, the fixed point of $S^{\prime \prime}=S_{2} S S_{2}$ in $P^{2}$. Then $L^{\prime}=T(L)$ is the line through $t$ and $s^{\prime}$, the fixed point of $S^{\prime}=S_{1} S S_{1}$. The part $R$ of $D^{*}(t)$ (see Corollary 15) bounded by $L$ and $L^{\prime}$ is a fundamental region of $G$ in $D^{*}$. If $T$ is hyperbolic, then the intersection of $R$ with the axis $L_{T}$ of $T$ is the fundamental domain of $\langle T\rangle$ on $L_{T}$. Hence $\pi\left(L_{T}\right)$ is simple in $M$.

Remarks. 1. Since $(T t, t)=(t, t)$, the conic $(T x-x, x)=0$ is degenerate. If $T$ is elliptic or parabolic, then the region $R$ in (34) coincides with $P^{2}-p_{t}$, where $p_{t}$ is the polar of $t$. If $T$ is hyperbolic, then $R$ contains $R_{T}$. In the latter case, for isotropic $w$, the geodesics in $H^{2}$, images of polars of indefinite points in $R$ under $\Phi$ (see Example 3), lie under the axis of $T$.

2. For the polars of indefinite points $f$ in (34) we have the following:

The geodesic $\gamma=\pi(L)$ in $M$ is simple if and only $L \cap T(L) \in D^{2}$. 


\section{REFERENCES}

[1] B.N. Apanasov, Discrete Groups in Space and Uniformization Problems, Kluwer Academic Publishers, Dordrecht/ Boston/London, 1991. MR 93h:57026

[2] A.F. Beardon, The Geometry of Discrete Groups, Springer-Verlag, New York, 1983. MR 85d:22026

[3] A.F. Beardon, J. Lehner, M. Sheingorn, Closed Geodesics on a Riemann surface with Application to the Markoff Spectrum, Trans. Amer. Math. Soc. 295 (1986), 635-647. MR 87g:11066

[4] J.W.S. Cassels, An introduction to Diophantine approximation, Cambridge Univ. Press, 1957. MR 19:396h

[5] J.W.S. Cassels, Rational Quadratic Forms, Ac. Press, NY, 1978. MR 80m:10019

[6] H. Cohn, Approach to Markoff's minimal forms through modular functions, Ann. of Math. (2) 61 (1955), 1-12. MR 16:801e

[7] H. Cohn, Representation of Markoff's binary forms by geodesics on perforated torus, Acta Arith. 18 (1971), 125-136. MR 44:5277

[8] H.S.M. Coxeter, The Real Projective Plane (2nd ed.), Cambridge University Press, London, 1960. MR 22:2929

[9] T.W. Cusick and M.E. Flahive, Markoff and Lagrange spectra, Math. Surveys and Monos., vol. 30, Amer. Math. Soc., Providence, R.I., 1989. MR 90i:11069

[10] H. Davenport and H. Heilbronn, On the minimum of a bilinear form, Quart. J. Math., Oxford Ser. 18 (1947), 107-121. MR 9:79c

[11] J. Elstrodt, F. Grunewald, and J. Mennicke, Arithmetic applications of the lattice point theorem, Proc. London Math. Soc. (3) 57 (1988), 239-283. MR 89g:11033

[12] D. S. Gorshkov, Lobachevsky geometry in connection with some problems in arithmetic, Zap. Nauchn. Sem. Leningrad. Otdel. Math. Inst. Steklov., 67 (1977), 39-85. (Transl. in Journal of Sov. Math. (1) 16 (1981), 788-820). MR 58:27802

[13] A. Haas, Diophantine approximation on hyperbolic Riemann surfaces, Acta Math. 156 (1986), 33-82. MR 87h:11063

[14] A. Haas, Length spectra as moduli for hyperbolic surfaces, Duke Math. J. 52 (1985), 923-934. MR 87e:58207

[15] A. Haas, Diophantine approximation on hyperbolic orbifolds, Duke Math. J. 56 (1988), 531547. MR 90c: 11031

[16] H. Helling, On the commensurability class of the rational modular group, J. London Math. Soc. (2) 2 (1970), 67-72. MR 43:3353

[17] C. G. Lekkerkerker, Geometry of Numbers, Amsterdam 1969. MR 42:5915

[18] J.Lehner, M. Sheingorn, Simple Closed Geodesics on $\Gamma(3)$ Arise from the Markoff Spectrum, Bull. Amer. Math. Soc. 11 (1984), 359-362. MR 86b:11033

[19] A. V. Malyshev, Markov and Lagrange spectra, Zap. Nauchn. Sem. Leningrad. Otdel. Math. Inst. Steklov., 67 (1977), 3-38. (Transl. in Journal of Sov. Math. (1) 16 (1981), 767-788). MR 56:267

[20] A. A. Markoff, Sur les formes binaires indéfinies, Math. Ann. 15 (1879), 381-409.

[21] P.J. Nicholls, Diophantine approximation via the modular group, J. London Math. Soc. (2) 17 (1978), 11-17. MR 58:16551

[22] G. Rosenberger, Uber die Diophantische Gleichung $a x^{2}+b y^{2}+c z^{2}=d x y z$, J. Reine Angen. Math. 305 (1979), 122-125. MR 80d:10030

[23] Asmus L. Schmidt, Minimum of quadratic forms with respect to Fuchsian groups. I, J. Reine Angew. Math. 286/287 (1976), 341-368. MR 56:15566

[24] Asmus L. Schmidt, Minimum of quadratic forms with respect to Fuchsian groups. II, J. Reine Angew. Math. 292 (1977), 109-114. MR 56:15567

[25] Asmus L. Schmidt, Minimum of quadratic forms with respect to Fuchsian groups. III, (preprint).

[26] C. Series, The Markoff spectrum in the Hecke group $G_{5}$, Proc. London Math. Soc. 57 (1988), 151-181. MR 89g:11056

[27] L.Ya. Vulakh, The Markov spectrum of imaginary quadratic field $\mathbf{Q}(i \sqrt{D})$, where $D \not \equiv 3$ (mod 4) (Russian), Vestnik Moskov. Univ. Ser. 1 Math. Meh. 26 (1971), no. 6, 32-41. MR 45:1847

[28] L.Ya. Vulakh, Diophantine closed sets (Russian), Trudy Moskov. Ins. Radiotehn., Electron. i Avtomat., 52 (1971), 27-31. MR 58:27816 
[29] L.Ya. Vulakh, The Diophantine equation $p_{1}^{2}+p_{2}^{2}+5 q^{2}=5 p_{1} p_{2} q$ and the Markov spectrum (Russian), Trudy Moskov. Ins. Radiotehn., Electron. i Avtomat., 57 (1972), 54-58.

[30] L.Ya. Vulakh, The Diophantine equation $p^{2}+2 q^{2}+3 r^{2}=6 p q r$ and the Markov spectrum (Russian), Trudy Moskov. Ins. Radiotehn., Electron. i Avtomat., 67 (1973), 105-112. MR 58:21957

[31] L.Ya. Vulakh, On Markov spectra on sublattices related to Diophantine equations (Russian), Trudy XXV Nauchn.-tehn. Konfer. Moskov. Ins. Radiotehn., Electron. i Avtomat., Sec. Mat. (1976), 16-21.

[32] L.Ya. Vulakh, Classification of Fuchsian subgroups of some Bianchi groups, Canad. Math. Bull. (3) 34 (1991), 417-422. MR 92i:11047

[33] L.Ya. Vulakh, Maximal Fuchsian Subgroups of Extended Bianchi Groups, in "Number Theory with an Emphasis on the Markoff Spectrum" (Provo, UT, 1991), 297-310, Lecture Notes in Pure and Appl. Math., 147, Dekker, New York, 1993. MR 94g:11028

[34] L.Ya. Vulakh, Higher dimensional analogues of Fuchsian subgroups of PSL(2,o), Trans. Amer. Math. Soc. 337 (1993), 947-963. MR 93h:20055

[35] L.Ya. Vulakh, Diophantine approximation on Bianchi groups, J. Number Theory, 54 (1995), 73-80. MR 96g:11076

[36] L.Ya. Vulakh, Diophantine approximation in $\mathbf{R}^{n}$, Trans. Amer. Math. Soc. 347 (1995), 573-585. MR 95e:11076

[37] L.Ya. Vulakh, On Hurwitz constants for Fuchsian groups, Canad. J. Math., 49, (1997), 405416. MR 98a:11087

[38] L.Ya. Vulakh, Farey polytopes and continued fractions associated with discrete hyperbolic groups, Trans. Amer. Math. Soc. 351 (1999), 2295-2323. MR 99i:11054

[39] L.Ya. Vulakh, The Markov spectrum for triangle groups, J. Number Theory, (67), (1997), 11-28. MR 99e:11093

Department of Mathematics, The Cooper Union, 51 Astor Place, New York, New YORK 10003

E-mail address: vulakh@cooper.edu 